\title{
Civilisations
}

Revue internationale d'anthropologie et de sciences

humaines

$53 \mid 2005$

Musiques "populaires"

\section{French music, Cajun, Creole, Zydeco}

Ligne de couleur et hiérarchies sociales dans la musique francolouisianaise

\section{Sara Le Menestrel}

\section{(2) OpenEdition}

Journals

Édition électronique

URL : http://journals.openedition.org/civilisations/579

DOI : $10.4000 /$ civilisations. 579

ISSN : 2032-0442

\section{Éditeur}

Institut de sociologie de l'Université Libre de Bruxelles

\section{Édition imprimée}

Date de publication : 1 janvier 2006

Pagination : 119-147

ISBN : 2-87263-005-8

ISSN : 0009-8140

\section{Référence électronique}

Sara Le Menestrel, «French music, Cajun, Creole, Zydeco », Civilisations [En ligne], 53 | 2005, mis en ligne le 24 janvier 2009, consulté le 19 avril 2019. URL : http://journals.openedition.org/ civilisations/579; DOI : 10.4000/civilisations.579 


\section{French music, Cajun, Creole, Zydeco \\ Ligne de couleur et hiérarchies sociales dans la musique franco-louisianaise ${ }^{1}$}

\section{Sara LE MENESTREL}

Résumé : Ces dernières années, les artistes et intellectuels locaux ont multiplié leurs efforts pour faire valoir les collaborations étroites et les échanges entre Cadiens et Créoles dans le répertoire musical franco-louisianais. Cette représentation inclusive n'amoindrit pas la portée de certains clivages et d'identité diasporiques, même s'ils sont toujours combinés à d'autres critères d'appartenance. La ligne de couleur transparaît au travers des catégorisations musicales qui prévalent depuis la deuxième moitié du $20^{e}$ siècle et modèle les jugements musicaux actuels. Les clivages observés reposent également sur des hiérarchies sociales internes, issues notamment des multiples dimensions des identités créoles louisianaises. Les Cadiens et les Créoles partagent par ailleurs un sens aigu de la localité, combiné à une démarche commerciale inscrite dans une stratégie de reconnaissance.

Mots-clés : Louisiane, ligne de couleur, musique cadienne/cajun, musique créole, zydeco.

Summary : These past few years in Southwestern Louisiana, many artists and intellectuals have increasingly tried to emphasize the close collaborations and exchanges between Cajuns and Creoles within French music. Albeit inclusive, this representation of local music persist alongside social divisions and diasporic identities, while at the same time combining them with other relevant identity-building criteria. The color line appears through musical categories which have prevailed since the second half of the $20^{\text {th }}$ century and which have shaped the present judgements on music. These divisions are also based on internal social hierarchies given the multiple facets of Louisiana Creole identities. Nonetheless, Cajuns and Creoles share a strong sense of place combined with an appeal for commercial success which points to a strategy of recognition.

Keywords : Louisiana, color line, Creole music, Cajun music, Zydeco.

1. Cet article est fondé sur deux enquêtes de terrain effectuées dans la région de Lafayette, dans le SudOuest louisianais, en 2001, puis en 2003 grâce à une bourse Fulbright. Je tiens à remercier Kali Argyriadis, Véronique Boyer, Jacques Henry et Rebecca J. Scott pour leur lecture attentive et leurs suggestions, ainsi que Bruce Raeburn pour avoir nourri ma réflexion alors naissante sur le lien entre jazz et musique française. 
$\mathrm{E}$ tablie depuis les années 1970 dans le Sud-Ouest de la Louisiane, où elle s'est mariée à un accordéoniste cadien et a formé plusieurs groupes de musique cadienne, Ann Savoy a récemment produit deux $\mathrm{CD}$ qui rendent hommage à la musique franco-louisianaise : Evangeline Made est consacré à la musique cadienne, celle des «descendants des Acadiens, blancs et francophones », tandis que Creole Bred met à l'honneur «la musique zydeco et créole» des «Afro-Américains francophones »" Le choix des titres traduit bien la perception des Cadiens et des Créoles comme issus de deux diasporas : le premier revendique l'ascendance acadienne des Cadiens au travers du personnage emblématique d'Evangéline - héroïne du poème de Longfellow (1847) qui narre le périple des Acadiens déportés de Nouvelle-Ecosse par les Anglais en 1755. L'origine acadienne est ici privilégiée, parmi les multiples groupes dont les Cadiens sont issus (colons français et espagnols dits Créoles blancs ${ }^{3}$, immigrés français, irlandais, écossais et allemands); par le choix d'un terme inscrit dans le champ lexical de l'hérédité génétique (bred), le titre du deuxième CD met l'accent sur la dimension biologique de l'identité des Créoles, qui désignent ici les descendants de gens de couleur libres et d'esclaves. Alors que vingt ans auparavant, Ann Savoy publiait un livre sur la musique cadienne qui mettait en lumière l'étroite collaboration des musiciens cadiens et créoles dans la construction d'une tradition musicale « française » (Savoy, 1984), elle établit néanmoins des catégories musicales fondées sur les ascendances distinctes des deux groupes.

Parallèlement à ce souci de distinguer Cadiens et Créoles, ces dernières années, les artistes et intellectuels locaux ont multipliés leurs efforts pour valoriser les échanges entre les différents styles établis au sein de la tradition musicale régionale. Des enregistrements et des performances rassemblent ainsi musiciens cadiens et créoles et mettent en avant leurs influences mutuelles. Cette représentation inclusive des traditions musicales locales contraste néanmoins avec des pratiques et des discours sur la musique révélateurs de divisions sociales persistantes. Celles-ci sont fondées sur la combinaison de plusieurs facteurs : la ligne de couleur transparaît au travers des catégorisations qui prévalent depuis la deuxième moitié du $20^{\mathrm{e}}$ siècle, et modèle les jugements musicaux actuels. Les clivages observés reposent également sur des hiérarchies sociales internes, issues notamment de la complexité et des multiples dimensions des identités créoles louisianaises. Au sein de cette dynamique sociale divisée apparaissent toutefois des espaces de négociations qui révèlent l'impact de certaines valeurs communes aux Cadiens et aux Créoles.

2. Evangeline Made. A tribute to Cajun music, Vanguard Records, 2002; Creole Bred. A tribute to Creole \& Zydeco, Vanguard Records, 2004.

3. La polysémie du terme « créole » en Louisiane le rend très confus. A l'époque coloniale, il s'appliquait à tout ce qui provenait du territoire louisianais, personne, produit ou langue. Jusque dans les années 182030, il est donc dénué de toute distinction de couleur, de statut social ou d'origine ethnique. L'arrivée des Anglo-Américains au début du $19^{\mathrm{e}}$ siècle amène à une distinction entre Créole blancs et Créoles de couleur. Aujourd'hui, dans la région de Lafayette, «Créole » se réfère aux descendants des gens de couleur libre et des esclaves émancipés ( $c f$ Le Menestrel, 2005, à paraître). 


\section{La musique française ${ }^{4}$}

Avant les années 1960 prédominait une perception inclusive de la musique francolouisianaise, sans distinction entre musique cadienne, musique créole et zydeco. On parlait alors en français de musique française ou en anglais de French Music, cette catégorie englobant une diversité de styles sans l'associer à un groupe spécifique, par contraste avec la terminologie actuelle qui parle désormais de musique cadienne et musique créole selon l'origine des musiciens. Le clivage français/américain était alors le plus prégnant, comme en témoigne de façon lumineuse une Cadienne qui grandit dans les années 60 : étant enfant, il ne faisait pour elle aucun doute que les mentions AM/FM de son poste radiophonique se référaient à la distinction American Music/French Music.

$\mathrm{Au}$ début du $20^{\mathrm{e}}$ siècle, les premiers enregistrements franco-louisianais témoignent d'une tradition musicale commune. Devenus les figures emblématiques de cet héritage, l'accordéoniste créole Amédée Ardoin (1896-1941) et le violoniste cadien Denis McGee (1893-1989) ont enregistré un album en 1929. Tous deux métayers, leur duo se poursuivit vingt années durant et remporta un immense succès dans les salles de danse locales et les bals de maison, inspirant jusqu'à aujourd'hui des générations de musiciens. Amédée Ardoin est aujourd'hui considéré comme une référence incontournable par les amateurs de musique franco-louisianaise, tous styles confondus.

Tout au long du siècle, cette musique se nourrit d'influences très diverses. Jusqu'au deux-tiers du $19^{\mathrm{e}}$, le violon en constitue l'instrument principal, souvent utilisé par pair, l'un jouant la mélodie, l'autre « secondant» en jouant l'harmonie ou un bourdon. Puis l'accordéon diatonique prend progressivement le devant de la scène, accompagné d'une section rythmique composée d'une guitare et de percussions ('tit fer et/ou frottoir). Dans les années 30, l'influence du western swing met à l'honneur les string bands. L'amplification conduit notamment à l'introduction de la steel guitar, qui fait désormais partie intégrante de l'instrumentation, tandis que le violon est accompagné d'autres instruments mélodiques comme le banjo et la mandoline. L'anglais est utilisé dans bien des chansons, d'autres sont traduites en français. Parmi les groupes les plus populaires du milieu des années 1930 tels Leo Soileau, the Rayne-Bo Ramblers et plus tard Harry Choates, the Hackberry Ramblers puise dans les répertoires aussi variés que celui de Bob Wills, Jimmie Rogers et Bessie Smith. Leader du groupe, Lurderin Darbone explique qu'il combina morceaux cadiens, hillbilly ainsi que ragtime, fox-trot et jazz. Le répertoire français n'était ainsi jamais exclusif d'autres styles : Joe Falcon, le premier à enregistrer un album en 1928, incluait dans ses concerts la variété de l'époque. Canray Fontenot, violoniste créole qui joua des années 40 jusqu'à sa mort en 1995, connaissait quant à lui de nombreux rags. De façon générale, dans la première moitié du $20^{\mathrm{e}}$ siècle, les groupes de cette région étaient tenus, s'ils voulaient emporter l'adhésion du public, d'interpréter la musique "populaire» de l'époque, entendue alors comme l'ensemble des airs qui étaient en vogue.

Parmi les différents emprunts constitutifs de la musique française d'alors, le jazz occupe une place privilégiée en dépit du peu d'intérêt porté par les chercheurs à ce lien, puisque seul un petit ouvrage lui a été jusqu'à présent consacré (Sonnier, 1989).

4. Il ne s'agit pas ici de retracer l'histoire de la musique du Sud-Ouest louisianais, mais de souligner la diversité de ses influences. Pour plus de détails, voir Ancelet, 1989; Bernard, 1996; Broven, 1983; Sandmel et Olivier, 1999; Savoy, 1984; Tisserand, 1998. 
Présentés comme deux styles distincts, le jazz de La Nouvelle-Orléans et la musique franco-louisianaise du " pays cadien » (dans le Sud-Ouest louisianais) semblent associés respectivement à une Louisiane urbaine et rurale sans lien entre elles. Les entretiens menés dans la région de Lafayette confirment une absence très fréquente de référence au jazz lorsque sont évoquées les salles de danse et les clubs de la première moitié du $20^{\mathrm{e}}$ siècle. Pourtant, dès les premières décennies du siècle se développa un cercle de musiciens de jazz prééminents dans les petites communautés rurales autour de Lafayette. Les échanges mutuels avec La Nouvelle-Orléans étaient incessants par le biais de séjours, de tournées ou d'engagements de musiciens. Plus d'une quinzaine d'orchestres et de brass bands tournaient plusieurs fois par semaine, le plus souvent dans les clubs, les parades et les fêtes paroissiales, attirant régulièrement des musiciens de la Nouvelle-Orléans - parmi lesquels Louis Armstrong, Count Basie et Cab Calloway - pour jouer rags, blues, foxtrot, polkas, mazurkas, charleston et valses, selon le public ${ }^{5}$.

$\mathrm{Au}$ lendemain de la seconde guerre mondiale, un autre genre musical va se développer chez les Cadiens comme les Créoles : le swamp pop ${ }^{6}$. Défini le plus souvent comme un hybride issu du rythm and blues de La Nouvelle-Orléans, du country and western, et de la musique cadienne et créole, ce style a émergé dans le Sud-Est du Texas et le SudOuest de la Louisiane, marquant durablement le répertoire franco-louisianais (Bernard, 1996). C'est à la même époque que le zydeco est popularisé, devenant indissociable des Créoles noirs. Inspiré de la musique française, du blues et du rythm and blues, l'orchestre zydeco se compose le plus souvent d'un accordéon piano chromatique, ou/et diatonique, accompagné d'une forte section rythmique où le frottoir joue un rôle prépondérant, et sans violon, contrairement au style cadien où la dimension mélodique est privilégiée. Même si le zydeco est le fruit d'une longue maturation, c'est à Clifton $\mathrm{C}$. Chenier que l'on attribue le mérite de l'avoir propulsé sur la scène nationale avec la chanson Les $z$-haricots sont pas salés (évoquant les temps durs, pendant lesquels on n'avait pas de viande - conservée dans le sel - pour accompagner les haricots) ${ }^{7}$. L'origine du terme zydeco est bien souvent attribuée à ce titre, bien qu'elle semble dépasser le cadre louisianais ${ }^{8}$. L'expression « les haricots sont pas salés » précède d'ailleurs cette période puisqu'elle apparaît dans les enregistrements des Lomax de 1934'. Chenier emploie ce terme comme style musical (mais aussi lieu de danse et style de danse) à partir de 1955 ,

5. Bunk Johnson fut membre aux côtés de George Lewis du groupe de jazz le plus important de la région, le Banner Orchestra, qui tourna pendant près de 40 ans. Né à La Nouvelle-Orléans en 1880, il s'installe à New Iberia (au sud-est de Lafayette) en 1920 après une carrière aux côtés de Jelly Roll Morton, Joe 'King' Oliver, et dans l'orchestre de Buddy Bolden, parmi d'autres. Il tourne avec plusieurs groupes à l'échelle locale, nationale et internationale, enseigne la musique et la trompette dans les établissements scolaires public de New Iberia, laissant ainsi son empreinte sur plusieurs générations d'étudiants.

6. Parmi les grands succès de ce style figurent Mathilda (du groupe Cookie and the Cupcakes) et See you later, Alligator (Bobby Charles Guidry).

7. Par extension, cette expression a pris une signification générale, répondant à la question : « Comment va les z-haricots? » [Comment ça va ?].

8. Se basant sur les travaux d'un ethnomusicologue français, B. Ancelet note que les chansons sur le thème des haricots sont partie intégrante d'une tradition musicale des îles créolophones de l'Océan indien, le séga zarico, de formation comparable au zydeco (1996 : 129). Les haricots évoquent ici des relations amoureuses malheureuses ou une sexualité frustrée.

9. Il s'agit de deux jurés, connues sous le nom de Dégo et J'ai fait tout le tour du pays (Cajun \& Creole music II, Rounder Records, 1999). 
dans l'album Zydeco Stomp. Mais le zydeco devient un genre nommé comme tel et réputé au-delà du Sud-Ouest louisianais à partir du moment où la maison de production Arhoolie enregistre C.C. Chenier en 1964.

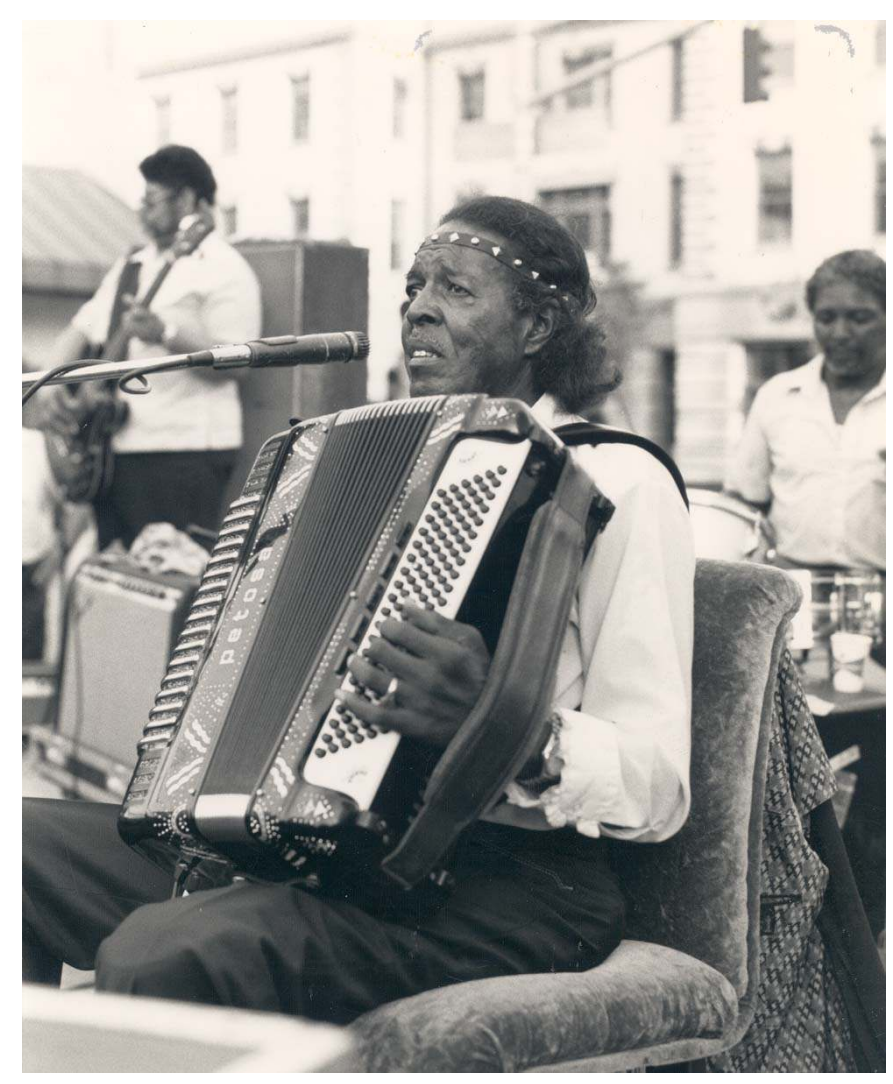

Photo 1 : Clifton Chenier (1925-1987), Lafayette, 1981 ( Jacques Henry).

Le répertoire franco-louisianais a donc toujours été très éclectique, nourri d'influences multiples et donnant lieu à l'émergence parallèle de plusieurs styles qui se sont inspirés de ces différents apports, par contraste avec l'image d'un âge d'or évoqué par certains pour appuyer leur perception de la musique « traditionnelle». Les appréciations des amateurs de cadien et de zydeco sont de surcroît elles-mêmes le fruit d'une évolution, leur goût ayant été modelé par différentes périodes successives. Jusqu'au renouveau musical des années 70, l'attrait des Louisianais (public et musiciens) pour la musique cadienne se prononce graduellement, après une immersion quasi-systématique dans le rock'n roll, le blues, le country and western et le swamp pop. 


\section{La revendication d'une tradition d'inclusion}

Depuis ces dix dernières années, le milieu artistique et intellectuel local insiste tout particulièrement sur la mixité culturelle dont la musique franco-louisianaise est issue, dans une volonté de mettre en avant l'héritage commun des Cadiens et des Créoles, leurs échanges et leur interaction, et de transcender ainsi la ligne de couleur. Les jeunes groupes cadiens sont très explicites sur ce sujet et tiennent à exposer cette perception à leur public. Le groupe Balfa Toujours, mené par la fille du célèbre violoniste Dewey Balfa, pilier du renouveau musical des années 1970, revendique dans la plupart des jaquettes de ses albums la tradition d'inclusion des Cadiens, en l'inscrivant dans leur histoire : «Leur culture en expansion fut renforcée par les influences externes, en les adaptant à la sensibilité cadienne. C'est inclusion, et non l'exclusion, qui a fait perdurer la culture cadienne à travers les siècles $\rangle^{10}$. Comme d'autres artistes et universitaires, le groupe combine systématiquement dans ses textes la valorisation de la mixité culturelle à l'hommage rendu à leur résistance à l'adversité. La tradition d'inclusion apparaît ainsi comme une condition sine qua non de la survivance des Cadiens malgré les souffrances endurées au fil des siècles (déportation par les Anglais, stigmatisation comme white trash, interdiction de parler français...).

Christine Balfa met un point d'honneur à perpétuer cette collaboration : elle a créé en 2001 le Dewey Balfa Cajun \& Creole Heritage Week. Ce stage musical s'applique à mettre à l'honneur l'héritage commun des Cadiens et Créoles, dépassant la division entre style cajun «blanc» et style zydeco «noir ». De vieux musiciens sont invités à jouer l'ancien répertoire « français » et évoquent l'interaction entre les deux groupes dans leur récit de vie. Présentant le violoniste créole Rodney Fontenot, Ch. Balfa évoque ses liens de parenté avec lui et ajoute avec conviction qu'elle en est « fière », tenant à transmettre ce message au public majoritairement non-louisianais du stage. On retrouve chez Barry Ancelet, universitaire spécialiste des Franco-Louisianais et actif militant culturel, un souci semblable : la musique d'un groupe de Créoles établis en Californie (les Frères Poulard) lui paraît tout autant inspirée par les grandes figures que sont Iry Lejeune and Aldus Roger [Cadiens] que par Amédée Ardoin and Adam Fontenot [Créoles], comme il l'explique dans le livret du CD : «Il aurait été facile de suivre les leçons exclusives d'un passé qui a trop souvent séparé les gens. Les Poulard et Garnier nous offrent un exemple d'inclusion d'autant plus élégant ». Don Cravins, Créole très renommé à la fois pour ses actions en faveur du zydeco (auquel il a consacré des émissions radio, télévisées et un festival extrêmement populaires) et sa fonction de sénateur louisianais, considère quant à lui le répertoire français comme "le blues des Cadiens et des Créoles 》, en référence aux souffrances et à la misère qu'elle évoque et qui crée entre eux ce qu'il perçoit comme une "parenté ».

Le discours revendicatif des non-Louisianais établis dans la région depuis plusieurs années, diffère quelque peu de celui des Louisianais : si ces derniers peuvent partager la même volonté de condamner une vision « racialisée » de la culture franco-louisianaise, ils n'en reconnaissent pas moins les limites des échanges entre Cadiens et Créoles. Tandis que Dirk Powell, du groupe Balfa Toujours (cf. photo de couverture), oppose la situation contemporaine aux liens étroits qui unissaient Cadiens et Créoles par le passé, sa femme, Ch. Balfa, est consciente du caractère contextuel de l'ouverture d'esprit que manifestent

10. A vieille Terre haute, 1995, Swallow Records; La Pointe, 1998, Rounder Records. 
certains musiciens à leurs côtés, sans que cela corresponde toujours à leur véritable point de vue. Elle remarque d'autre part que son propre père, fort respectueux du rôle des Créoles dans la musique dite cadienne, n'a pourtant jamais joué avec 'Bois Sec' Ardoin accordéoniste créole et neveu d'Amédée Ardoin - qu'il côtoyait bien souvent. Le respect mutuel n'est pas confondu avec la sociabilité. Le contraste est d'autant plus grand avec les communautés de fans établies aux Etats-Unis et dans le monde, qui entretiennent bien souvent l'image d'une musique fédératrice et organisent systématiquement des festivals, concerts et stages où musique cadienne et zydeco sont célébrés de concert, si bien que ses amateurs se sont baptisés explicitement des «CZ fans ».

Si ce n'est par le discours, la conception syncrétique de la culture franco-louisianaise est exprimée au travers de chansons ou des choix musicaux des groupes. Balfa Toujours adopte cette stratégie avec la chanson Un ange pour tout de la Louisiane, introduite par un texte sans ambiguïté : "Qu'on le veuille ou non, les cultures de cette région sont redevables les unes aux autres de leur survie. Cette chanson tient à rappeler que nous ne sommes pas aussi différents que certains aimeraient nous le faire croire $\rangle^{11}$. Horace Trahan, jeune accordéoniste cadien considéré par ses fans comme l'héritier direct du style d'Iry Lejeune (musicien de renom dans les années 1940), n'hésita pas à rompre avec le style « traditionnel » qu'il incarnait pour former un groupe de zydeco avec des musiciens noirs. La chanson titre de son premier album, Reach Out and Touch a Hand, affiche sans détour ses convictions et vient d'emblée légitimer son choix ${ }^{12}$. Plusieurs groupes cadiens de renom incorporent de plus en plus souvent dans leur répertoire des morceaux de musiciens créoles ou du zydeco : Beausoleil donna le ton au début des années 1980 avec l'album Zydeco Gris-gris, et continue de mettre en avant des inspirations multiples (zydeco, country, blues, latino, jazz, tex-mex ...) réinterprétées dans le style cadien, comme l'explicite le titre de l'album Cajunization (1999). Balfa Toujours a enregistré un album avec 'Bois Sec' Ardoin; Steve Riley and The Mamou Playboys reprend depuis plusieurs années des succès de C.C. Chenier et des airs de swamp pop. Il a en outre incorporé des extraits de juré des années 30 (musique sans instruments, avec mains, pieds et voix) dans une chanson rock très contemporaine, et a mis en musique un poème qui aurait été écrit en créole par un esclave en 1860. La portée de ces choix est d'autant plus grande qu'elle fait écho à des perceptions musicales divergentes, témoignant de la prégnance de certains clivages sociaux ancrés dans l'histoire des Franco-Louisianais.

11. La Pointe, Rounder Records, 1998.

12. Reach out and touch a hand (x3)/And make a friend if you can/It doesn 't matter if you're Black/It doesn't matter if you're White/It doesn't matter who you are/In the eyes of the Lord" (Horace Trahan and the New Ossun Express, Fred Charlie/Zydeco Hound Records, 2001). 


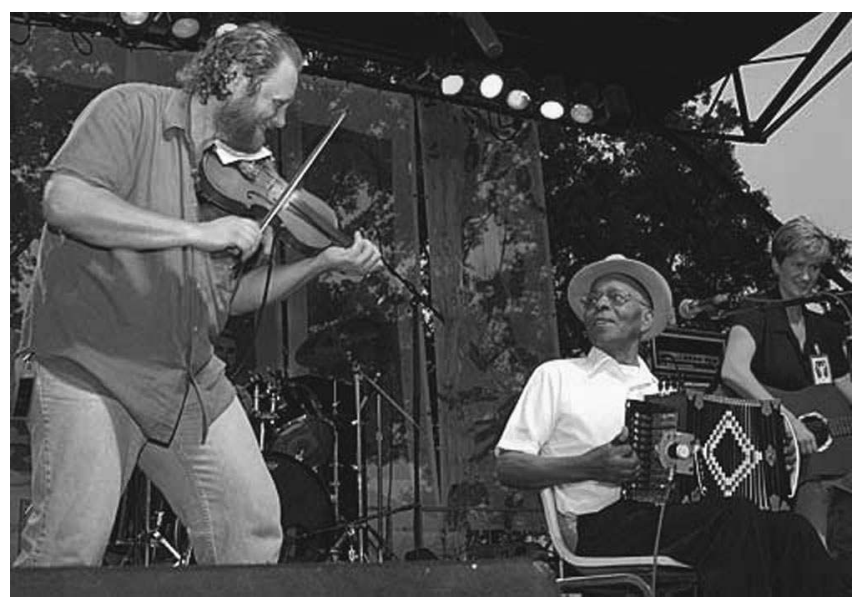

Photo 2: 'Bois sec'Ardoin et Kevin Wimmer, violoniste du groupe Balfa Toujours, Dewey Balfa Cajun \& Creole Heritage Week, Lake Fausse Pointe, 2001

(C) David Simpson, LSUE Office of Public Relations).

\section{Hiérarchies sociales et discours de réappropriation parmi les Créoles}

Contextuelles et multi-dimensionnelles, les représentations identitaires des Créoles se déclinent en différentes combinaisons : c'est ainsi que dans le Sud-Ouest de la Louisiane, l'identité créole n'interfère pas avec un fort sentiment d'appartenance aux Africains Américains, si ce n'est dans quelques familles issues d'anciennes enclaves créoles rurales ${ }^{13}$. Identité noire et héritage français sont revendiqués de concert. Beaucoup soulignent la discrimination qu'ils ont subi avec les Noirs, quelle que soit leur teinte de peau, et rappellent que pendant la ségrégation, que l'on soit clair ou foncé, "on connaissait sa place ». Par contraste, à La Nouvelle-Orléans, mais aussi dans la région de Natchitoches (au Nord-Ouest) ainsi que parmi la diaspora créole (notamment californienne) persiste souvent un sentiment d'appartenir à une culture spécifique, issue d'un mélange unique entre Européens, Africains et Amérindiens, lié à un refus d'être identifié comme Noir ou Blanc. Il semble que de manière générale l'origine rurale accroisse la primauté accordée à l'identité créole, bien que cette tendance soit également présente chez les Néo-Orléanais.

Ces perceptions concurrentes, qui conduisent à des accusations d'élitisme, renvoient à la catégorie des gens de couleur libres en Louisiane, insérés dans une société louisianaise tripartite qui leur donna les moyens de posséder des biens (parmi lesquels des esclaves) et de les transmettre ${ }^{14}$. Cette distinction créa chez certains une conscience de classe qui les incita à s'identifier comme Créoles de couleur après la guerre de Sécession, afin de se distinguer des esclaves émancipés auxquels ils furent assimilés pendant la ségrégation ${ }^{15}$.

13. Les termes «Africains Américains» ou «Noirs» font référence aux catégories employées par ces personnes.

14. Pour plus de détails sur ces différentes perceptions, $c f$. Le Menestrel, 2005 (à paraître).

15. Certains Créoles de couleur n'en en pas moins joué un rôle déterminant dans la défense de l'égalité des droits des Noirs pendant la ségrégation : parmi d'autres figures militantes, L. Martinet et R. Desdunes ont ainsi fondé le Comité des citoyens pour dénoncer comme une violation d'un droit constitutionnel la législation du Separate Car Act de 1890 mise en application dans le procès Plessy v. Ferguson. 
Aux alliances endogames s'ajoutent d'autres stratégies de distinction. La famille, la langue, la couleur et certaines valeurs morales constituent des facteurs déterminants dans le maintien d'une identité distincte (Anthony, 1978 : 165).

Dans la région de Lafayette, même les Créoles les plus revendicatifs réfutent la prégnance de la classe comme pilier de leur identité en lui substituant celle de la culture : "We are Creole Black versus African American Black», affirment-ils en insistant ainsi sur la compatibilité entre l'identité créole et noire. Il arrive pourtant que même lorsque l'identité noire est revendiquée, l'ascendance africaine ne le soit pas : "C'était [un mélange d'] Indiens et d'Africains, de Français et d'Indien. Mais jamais de Français et d'Africains. Les Français n'ont jamais pris d'Africaines pour femmes. Femme jolie [en français dans le texte]. Ils voulaient les belles femmes mulâtres ».

Jim, 63 ans, est catégorique ${ }^{16}$ : les Créoles ne furent jamais descendants d'esclaves, et ne furent pas non plus le fruit d'unions illégitimes: "Ce n'était pas simplement sexuel, c'était un engagement, un engagement à vie " (en référence au plaçage ${ }^{17}$ ). Tous ses arguments consistent à occulter une ascendance africaine dévalorisée pour que la supériorité de leur statut social soit indiscutable : "Les Créoles étaient cultivés, ils étaient raffinés ». Cette perception élitiste va de pair avec la volonté de jouer un rôle de leader politique - comme s'y appliquèrent les Créoles pendant la Reconstruction. Jim s'inscrit dans cette même perspective de leadership dans le domaine de l'éducation, ayant consacré sa carrière à promouvoir l'accès aux études supérieures en dirigeant plusieurs programmes éducatifs louisianais de soutien et d'aides. Son souci de magnifier les réalisations des Créoles et de leur attribuer un rôle prépondérant dans l'éducation, l'émancipation et la défense des droits civiques des Noirs louisianais s'inscrit dans une histoire officielle célébrative qui contraste avec une mémoire collective et individuelle conflictuelle et tourmentée (Thompson, 2001).

L'enjeu de pouvoir que constitue aujourd'hui le zydeco amène Jim à tenir un discours de réappropriation. La distinction sociale que certains membres de l'élite créole persistent à maintenir aboutie à une opposition supplémentaire entre descendants d'esclaves et de Créoles de couleur, les uns s'inscrivant selon Jim dans une démarche commerciale, les autres sans prétention à la renommée, implicitement dépréciée.

"Le zydeco a vraiment commencé avec les Créoles (...). Les Créoles n'ont jamais voulu diffusé leur culture. Et un type comme Clifton Chenier en a profité. Il a commencé à l'amener à une communauté plus large. Il a pris la musique créole et l'a appelé zydeco. N'oublie pas que Chenier, ses fans, beaucoup de leurs ancêtres étaient esclaves de Créoles. Certains Créoles se sont par la suite mariés avec des esclaves. Et le mélange a commencé ».

Les Créoles de couleur auraient ainsi transmis leur héritage musical à leurs esclaves, la figure emblématique du zydeco leur étant elle-même redevable de son succès. Or, les Créoles regroupent depuis le début du siècle des descendants de Créoles de couleur

16. Les pages indiqués datent de 2003.

17. Le plaçage était une forme institutionnalisée du concubinage par lequel une femme de couleur libre négociait son union avec un homme blanc tenu par contrat de la prendre en charge financièrement ainsi que leurs enfants. Tandis que Jim perçoit cette pratique comme valorisante, nombre d'intellectuels créoles dénoncèrent dès le milieu du $19^{\mathrm{e}}$ cette pratique qu'ils jugeaient dégradante et prônèrent des alliances endogames (Bell, 1997). 
libres mais aussi des descendants d'esclaves. En outre, Clifton Chenier était précisément issu d'une famille de Créoles de couleur. Que cette ascendance lui soit déniée ou soit simplement jugée inconcevable semble relever de l'association souvent tacite entre identité créole et clarté de peau. Animateur d'une émission de radio sur le zydeco, John ne restreint pas l'origine du zydeco aux Créoles, mais son discours illustre bien cette perception :

* «Un Créole, ou un mulâtre, c'était quelqu'un qu'était manière brun avec des bons cheveux, des cheveux droits, des yeux manière bleus, gris, parce que mon père c'était un créole (...). Un monde noir il aime la musique de zydeco aussite. Qui c'est qui joue asteur le zydeco ? Quand ça a commencé avec Clifton, il était un noir, un nègre, c'est tout, c'est pas un créole ${ }^{18} »$.

Il est également possible que cet usage du terme « Créole » en référence à la couleur exprime le refus d'accorder aux Créoles qui y prétendent un statut d'élite sociale. John se désolidariserait de la sorte de ceux qui, comme Jim, tiennent à être distingués des descendants d'esclaves et mettent en avant leur supériorité ${ }^{19}$. L'élite créole (propriétaires terriens et élite urbaine) inclut d'ailleurs dans cette distinction les Cadiens, soulignant leur statut social inférieur, leur ascendance paysanne et leur moindre niveau d'éducation.

\section{"The right color of music"}

"*Moi j’ai jamais compris la différence de la musique créole et la musique français, except que c'est un homme blanc qui joue l'accordéon quand c'est cadien, et c'est un homme noir quand c'est créole ».

Les catégorisations aujourd'hui employées pour désigner le répertoire français du début du siècle dernier laissent Fred, Cadien, producteur de disques, quelque peu perplexe. La musique n'est pas la seule concernée par cette pratique, appliquée aussi aux différentes formes linguistiques francophones - le créole et le français régional louisianais ${ }^{20}$ - qui peuvent être maîtrisés par une même personne quelle que soit son origine. Tandis qu'une majorité de Cadiens et de Créoles parlent le français régional, les Cadiens désignent leur langue comme du cadien, et les Créoles comme du créole. C'est ainsi le groupe d'appartenance revendiqué par le locuteur qui détermine le sens du terme employé et non la nature de la langue.

Ce mécanisme est particulièrement révélateur de la prégnance de la ligne de couleur dans les relations sociales et de son impact sur les représentations de la musique. Dennis,

18. Les extraits d'entretiens précédés d'un astérisque sont du français régional louisianais, les autres sont des traductions de l'anglais. Selon Brasseaux, (1994), 70 à 80\% des esclaves étaient désignés comme Noirs tandis que la quasi totalité des Créoles de couleurs étaient « mulâtres » avant la guerre de Sécession, à l'exception de la paroisse St Landry, d'où est précisément originaire C. C. Chenier. Par la suite, les intermariages ont amoindri ce facteur de distinction.

19. Cette association entre clarté de peau et élite sociale reflète d'ailleurs les statistiques de 2000, selon lesquelles les Noirs plus clairs de peau ont un statut socio-économique plus élevé, se marient avec des partenaires dont le statut est équivalent et ont une conscience identitaire « noire » moindre que les Noirs plus foncés. Cette corrélation n'a pas changé entre 1950 et 1980 (Hugues \& Hertel, 1990; Hill, 2000).

20. La désignation « français régional de Louisiane » a été proposée par T. Klingler (2004). 
musicien d'un groupe zydeco de renom, reconnaît bien les affinités musicales entre Cadiens et Créoles, mais il leur prête des styles bien distincts dont il explique le fondement :

"[Ardoin et McGee] étaient tous les deux d'excellents musiciens. J'adore leur musique. Mais si tu écoutes vraiment bien, ils ne jouent pas du tout la même chose. Je peux te dire la différence entre un violoniste noir et un violoniste blanc. C'est juste que tu peux me donner un peu de ton sang, mais il faut plus que ton sang pour que je sois comme toi. Il me faut tes os, ton cœur, ta sensibilité. Je peux entendre la subtilité de la musique. La subtilité est jouée avec le cœur (...). C'est comme si j'essayais de chanter comme un Indien. Je peux peut-être le copier, imiter une chanson parfaitement. Mais l'âme, la subtilité de l'âme...cet élément transparent ne peut pas être reproduit, ne peut pas être perçu, peut-être peut-il être senti. C'est difficile à expliquer, mais je peux sentir ça. Je sais quand c'est une âme sæur. Et je ne dis pas que c'est mieux, simplement c'est différent ».

Alors que le sang n'est pas jugé comme un critère suffisant pour transmettre la sensibilité et l'âme, qui fondent à ses yeux la différence, la couleur de peau, elle, est déterminante. L'impact du biologique sur le mental est tour à tour jugé inopérant et déterminant. Comme dans bien des sociétés, la revendication d'un "Black feel» impossible à acquérir totalement par un Blanc, et la représentation plus large d'un lien entre « race » et style musical, est très présente en Louisiane.

La distinction entre musiciens cadiens et créoles conduit en outre Dennis à conférer aux Créoles la paternité du style cadien, sans pour autant leur en laisser le monopole. Ce sont avant tout les esclaves qui auraient joué cette musique. Les Cadiens sont ici placés dans une relation de domination au même titre que les propriétaires d'esclaves (ceux qui occupaient le haut de l'échelle sociale, les Genteel Acadians, ont effectivement possédé des esclaves), qui incite à leur attribuer un rôle secondaire dans la construction de la tradition musicale, dont les Créoles apparaissent comme les principaux artisans ${ }^{21}$. La référence à l'Afrique vient par ailleurs fréquemment justifier la spécificité du style créole, même chez les Créoles qui refusent l'identification aux seuls Africains Américains et soulignent la mixité de leur ascendance : "La musique cadienne est différente de la musique créole. Le cadien avait un rythme français, un rythme français cassé. La différence, c'est que les Créoles avaient un rythme plus des Antilles, de style africain ».

La distinction entre style cadien et créole n'est par ailleurs pas toujours légitimée par l'origine et la couleur des musiciens. L'accordéoniste créole Joe Hall, qui joue avec le violoniste cadien Mitch Reed et revendique un style créole, explique :

" Si quelqu'un te dit que tu es un certain type de musicien à cause de ta couleur, c'est pas vrai. Regarde Mitch, c'est un des violonistes créoles les plus fort du coin (...). La différence entre le style cadien et créole? La musique est la même, c'est le style qui est différent. Ca peut être aussi simple que les paroles ou le pont [variation, ici de la seconde partie du thème, le B]. C'est pas quelque chose que tu

21. Sur le thème des appropriations réciproques de traditions entre Cadiens et Créoles, voir Le Menestrel, 1999. 
peux expliquer, c'est juste un style, c'est...Je peux ajouter mon propre B à chaque morceau que j'entends. Tu peux entendre l'accent européen. C'est vraiment ça le créole. Ça peut être [joué par] un Noir ou un Blanc mais c'est très lié à la dimension européenne ».

Cette dimension européenne que Joe Hall invoque sans vouloir plus l'expliciter légitime sa perception du style créole, "le plus pur [style] qu'on trouve ici». Les distinctions musicales, elles, demeurent pour lui du domaine de l'indicible, du ressenti, voire du cadre de vie. Le refus de faire de la couleur un critère distinctif doit par ailleurs être nuancé : seul son compagnon Mitch vient à ses yeux appuyer cette perception, tous les autres musiciens qu'il juge dignes interprètes du style créole sont noirs.

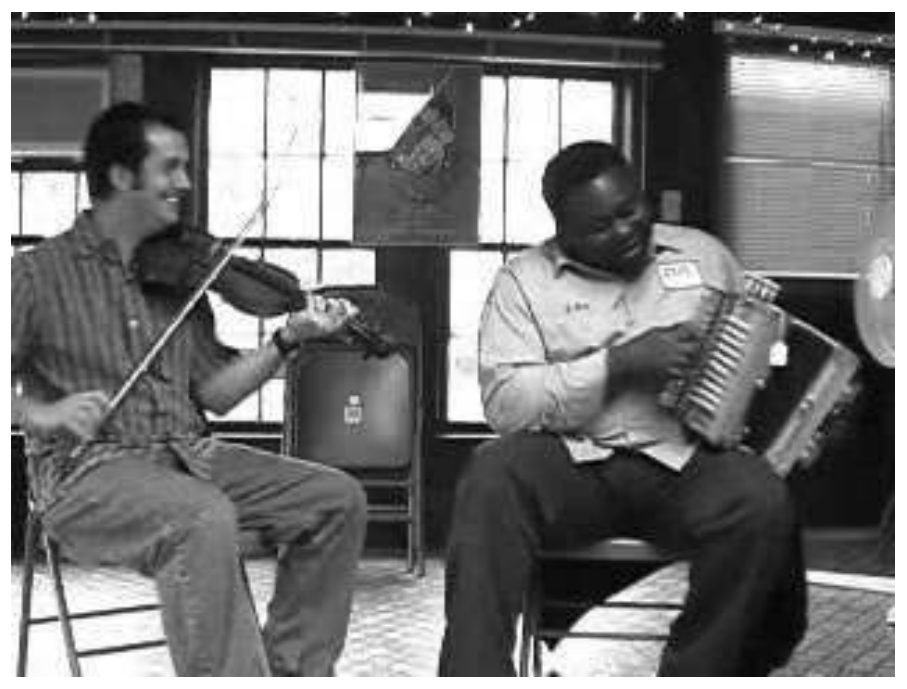

Photo 3 : L'accordéoniste Joe Hall et le violoniste Mitch Reed, Lafayette, novembre 2004

(C) Mark Guillory).

La distinction entre répertoire cadien et créole est souvent légitimée par les chercheurs (Broven, 1983; Tisserand, 1998; Wood, 2001). Ils se réfèrent souvent à une interview de Canray Fontenot dont le père, qui jouait avec Amédée Ardoin, racontait que ce dernier se rendait dans des « bals noirs » après avoir joué pour les « Blancs ». Il aurait alors joué un répertoire de blues et d'anciens chants africains. L'adaptation d'Ardoin au goût du public et sa polyvalence amènent ainsi certains à penser que ce qu'il jouait pour les Noirs était plus " authentique », fruit de sa propre créativité, par contraste avec la musique qu'il jouait avec et pour les Cadiens, qu'il n'aurait fait qu'interpréter. L. Back évoque à propos de la musique du Sud une logique similaire qui contribue à définir le blues, le rhythm and blues et la soul comme proprement « noirs »: «Quand les Blancs apprennent des Noirs, on y voit de l'imitation, mais quand les Noirs apprennent les mêmes accords, il s'agit automatiquement d'inspiration, dans laquelle on revendique leur héritage » (2002:230). Le souci de reconnaître le rôle des musiciens noirs dans le patrimoine musical américain conduit souvent à une vision « racialisée » des styles musicaux, qui fonde l'idée d'une 
propriété musicale, associée par W. B. Michaels à un «cultural geneticism » (cité dans Ware et Back, ibid.). En vertu de ce syndrome «Black through White », l'identification des Blancs à la musique noire est attribuée à un mélange de désir pernicieux et de fascination pour l'exotisme, et réduite à l'exploitation et à la récupération au détriment des Noirs.

La distinction entre style cadien et créole est également mise en avant par Sexton (2000 : 177), qui définit le style lala, autre dénomination du style créole (et des soirées dansantes), comme une « version du two-step plus syncopée d'influence africaine ». Dans la même lignée, Wood reconnaît les ressemblances des deux styles tout en affirmant leur différence - sans plus de précision - et en étendant cette influence africaine au zydeco (Wood, 2001: 25). De son côté, Bernard oppose le clivage entre cadien et zydeco au modèle «biracial » que représente le swamp pop : «Contrairement au genre cadien et zydeco, qui sont divisés selon une ligne raciale et fondés sur une instrumentation folk et des paroles francophones, le swamp pop est un genre biracial qui repose principalement sur des paroles anglophones et une instrumentation des années 1950 et de style rythm and blues. Il n'y a aucune différence entre les interprètes de swamp pop cadiens et les interprètes créoles. Leurs sonorités ne sont qu'une seule et même chose » (Bernard, 1996 : 8). La musique française persiste à être racialisée, opposée à un modèle d'hybridité incarnée ici par le swamp pop. Même lorsque le processus de créolisation est revendiqué comme caractéristique de la musique franco-louisianaise, la question de l'origine des styles qu'elle regroupe demeure l'objet de réappropriation. B. Ancelet précise que la chanson qui aurait inspirée celle de C.C. Chenier est d'origine acadienne (Hip et Taïau), de même qu'un juré de 1934 serait lui aussi «emprunté » au répertoire acadien (1996: 132). Au contraire, L. Post voit dans Hip et Tä̈au une «negro song », se fondant sur Joe Falcon, premier à l'avoir enregistrée, qui indique l'avoir apprise d'un métayer noir (cité dans Savoy, $1984: 95)$.

Il arrive par ailleurs que l'usage de l'accordéon par les Cadiens soit attribué aux Créoles, en faisant de l'instrument une "tradition d'origine afro-créole qui remonte au moins à $1850 »$ (Dole, 1995; Snyder, 1997 : 39). L'accordéon diatonique aurait été exporté d'Allemagne aux Etats-Unis à partir de 1840 et popularisé par les spectacles de ménestrels. Des maîtres en achetaient pour être divertis par leurs esclaves, ce qui nourrit la théorie d'un usage d'abord exclusivement par les Noirs. D'autres émettent la possibilité d'une utilisation parallèle chez les Cadiens (Minton, 1996 : 490). Les écrits locaux datent le plus souvent l'introduction de l'accordéon en Louisiane des années 1880, par le biais des immigrants allemands et de ceux du Midwest (Ancelet, 1989; Savoy, 1984).

L'évolution de la terminologie semblerait presque se faire en négatif de celle des relations sociales: tandis que pendant la ségrégation légale, on parlait de musique française, il semble qu'à partir des années 60 , dans le contexte du Mouvement des droits civiques, la perception de ce genre se trouve progressivement modelée par des frontières « ethniques », traduites par les appellations « musique cadienne » et « musique créole ». Cette distinction s'inscrit dans un climat politique d'essor du nationalisme noir, favorable à la revendication d'une légitimité culturelle fondée sur des critères " ethniques » et « raciaux ». Néanmoins, la prééminence d'une catégorie musicale plus inclusive dans la première moitié du $20^{\mathrm{e}}$ siècle ne reflète pas les relations sociales de l'époque, loin s'en faut. Le climat sociopolitique des années 20 et 30 est ainsi particulièrement tendu : le $\mathrm{Ku}$ Klux Klan ainsi que d'autres organisations racistes fleurissent à l'échelle nationale et locale, dans le Nord louisianais, tandis que la dépression économique exacerbe les 
discriminations raciales. Dans un tel contexte, les collaborations musicales entre Noirs et Blancs résultent de décisions et de choix individuels. Elles demeurent exceptionnelles, peu de musiciens étant disposés à prendre le risque de défier la ligne de couleur, qu'il s'agisse de musique française ou de jazz ${ }^{22}$.

Les circonstances de la mort d'A. Ardoin viennent illustrer la rigidité de la ligne de couleur à cette époque. Différentes versions circulent : pour certains, il se serait fait battre violemment tandis qu'il jouait dans une salle de danse pour avoir accepté des mains d'une fille blanche un mouchoir qu'elle lui avait tendu afin d'essuyer la sueur de son visage. D'autres, comme le musicien de zydeco Boozoo Chavis, sont persuadés qu'il n'aurait pas franchi aussi naïvement les frontières de la sociabilité, sachant fort bien que son rôle était circonscrit à celui d'un musicien, et pensent qu'il aurait plutôt été empoisonné par un Blanc; Denis McGee, lui (son partenaire), assure qu'il fut victime de la jalousie d'un Noir, accrue par sa collaboration avec un musicien blanc. Quoiqu'il en soit, Ardoin ne se remit jamais de ce dont il fut victime et se trouva interné dans un asile psychiatrique où il mourut en 1941.

Plus tard, dans les années 50, les musiciens de swamp pop créoles témoignent de relations très tendues : ceux qui se produisent dans des clubs cadiens doivent passer par la porte du fond, sont sommés de ne pas quitter la scène, sont escortés par la police pour se rendre au bar ou aux toilettes (Bernard, 1996 : 66). Une aventure avec une femme blanche vaut à Huey «Cookie » Thierry (leader de Cookie and the Cupcakes) d'être menacé par un shérif si bien qu'il finit par s'établir en Californie en 1965. La collaboration entre les musiciens de swamp pop cadiens et créoles existe bel et bien - le hit national This should go on forever de Rod Bernard s'inspire grandement d'une composition de King Carl - mais la version de ce dernier ne reçut que peu d'attention et les deux musiciens ne se produisirent jamais ensemble. La danse cristallise d'ailleurs bien souvent les phobies des ségrégationnistes, qui y voient un encouragement à «l'amalgamation raciale » (miscegenetion) $^{23}$. Chep Morrison, maire de La Nouvelle-Orléans dans les années 195060 , œuvra pour accroître les droits et les conditions de vie des Noirs, sans toutefois remettre en cause la ségrégation. Il accusa en 1960 son adversaire à la candidature de gouverneur de gérer « an integrated nightclub » à Palm Springs (Fairclough, 1995 : 179).

Le milieu musical reste aujourd'hui un espace privilégié pour étudier ces clivages. Les réactions à la conversion d'Horace Trahan en musicien zydeco sont à ce titre exemplaires. Sa décision d'abandonner son groupe cadien en 1999 est vécue comme une véritable trahison, un gâchis déploré aujourd'hui encore. La transition a été difficile et longue à faire admettre au public, noir comme blanc, et les clubs n'ont ouvert leur porte que très

22. A l'enregistrement d'Ardoin et McGee en 1929 fait écho celui de Sidney Arodin, clarinettiste blanc, avec Lee Collins, cornettiste noir, qui enregistrèrent la même année, à un mois d'intervalle, eux aussi à La Nouvelle-Orléans, avec The Jones and Collins Astoria Hot Eight. S'il semble qu'ils se soient par ailleurs produits ensemble, de telles collaborations parmi les musiciens de jazz du début du siècle se produisaient davantage en studio que sur scène où le risque était nécessairement plus grand. Ainsi, le New Orleans Rythm King, premier groupe de jazz à avoir enregistré un disque « racially mixed » en 1923, n'a pas fait de représentation avec Jelly Roll Morton, pourtant interprète dans l'album. (communication personnelle, Bruce Raeburn).

23. Cette menace est ressentie à l'échelle nationale : en 1943, le Bureau of Public Relations de l'armée américaine censure des photos de GI's noirs dansant avec des femmes britanniques parues dans plusieurs journaux américains, dont le magazine Life (Ware et Back, 2002 : 187). 
progressivement. Ses tentatives d'exprimer directement ses revendications aux danseurs ne trouvent guère d'écho. A l'Atchafalaya Club, à Henderson, Horace Trahan incorpore un jour entre deux chansons zydeco une chanson de Bob Marley - Judge Not - interprétée en solo à la guitare, en réponse aux bruits qui courent sur son abus d'alcool et de drogue. La piste de danse se vide aussitôt. Même ceux qui ne réprouvent pas son choix se désolent qu'il ne mette plus en valeur sa voix et ne s'entoure pas des meilleurs musiciens à leur goût, légitimant leur déception en lui donnant un fondement proprement musical.

Selon une autre dynamique, les Viator ont eux aussi été confrontés à des obstacles en raison de leurs choix. Alida, 19 ans, et son frère Moïse, 22 ans, jouent ensemble depuis leur enfance. En 2000, ils décident de former un groupe, Et Là-bas, dans lequel ils explorent le répertoire non seulement cadien mais aussi zydeco et le jazz créole de La Nouvelle-Orléans, s’entourant de musiciens cadiens et créoles. Le « Cajun French Music Association » (CFMA), qui n'hésite pas à exclure de ses concours les musiciens trop « progressistes » à ses yeux, reconnaît les talents de violoniste d'Alida et lui offre un stage de musique. Mais lorsque celle-ci choisit comme enseignant Mitch Reed, féru de l'ancien répertoire français, l'association refuse en rétorquant qu'il joue de la «Black music » et non du cadien, lui préférant un musicien influencé par le country and western. Le père des Viator explicite d'avantage cette perception : les personnes nées avant les années 30 étaient avant tout exposées à la musique de McGee et d'Ardoin. "Donc ils ne sont pas rebutés par les vieilles chansons créoles, pour eux c'est de la musique française. Ils ne traçaient pas une frontière ». Par contraste, les générations suivantes, elles, s'abreuvent d'une musique française fortement influencée par le country and western. L'écoute de l'ancien répertoire et de ses interprètes actuels les amène donc à l'associer à de "la musique noire ». Les différentes influences musicales au cours des décennies amènent ainsi à des représentations différentes selon les générations, conditionnées par le style le plus en vogue à leur époque.

Une seconde expérience confirme aux Viator la source des désapprobations de leur style : autrefois invités au Liberty Theater, à Eunice, au spectacle musical radiophonique « Le Rendez-vous des Cajuns », ils trouvent un jour porte close : "Ils ont dit qu'ils ne voulaient pas qu'ils jouent là, parce que leur musique n'est pas appropriée. Ils y ont joué pendant huit ans, ne sont jamais sorti sans ovation, et maintenant ils ne les invitent plus parce qu'ils ne jouent pas la bonne couleur de musique ", affirme leur père outré mais combatif, défendant par ailleurs sans relâche la langue créole qu'il parle plus volontiers que le cadien, rappelant ainsi qu'à l'image des dialectes qui ne sont pas exclusifs, les traditions musicales des Créoles et des Cadiens ne sont pas réductibles à l'un ou l'autre groupe.

Le style joué par les musiciens et leur couleur de peau entraînent systématiquement des conséquences sur la composition du public et leurs appréciations. Horace Trahan attire ainsi une majorité de Blancs dans les clubs de zydeco. La logique n'est pourtant pas identique avec Geno Delafosse, accordéoniste créole friand de l'ancien répertoire français. Comme pour Horace Trahan, Geno doit essuyer les condamnations de certains Créoles qui l'accusent de «jouer du cadien ». Très apprécié parmi les Cadiens, il attire un public majoritairement blanc. Danseur invétéré, enseignant ponctuel dans les stages de musique en dehors de la Louisiane, Don ne s'est rendu pour la première fois dans un club de zydeco que récemment, pour écouter Geno, qu'il connaissait personnellement. Il tente d'expliquer ses réticences : «J'ai tendance à aller dans les clubs où je me sens à l'aise. 
Surtout parce que je ne sais pas ce que je ferais si j'étais confronté par une personne d'une autre culture qui ne voudrait pas vraiment de moi là-bas ». La confusion culture/ couleur de peau légitime fréquemment la ligne de couleur. La supposition qu'on ne sera pas le bienvenu dissuade beaucoup de Cadiens de fréquenter les clubs zydeco, tandis que d'autres se justifient en défendant le droit des Créoles à avoir leur propre espace de sociabilité. Certains propriétaires de clubs zydeco ont d'ailleurs réservé une journée dans la semaine à des groupes zydeco «blancs ». C'est le cas de Hamilton's à Lafayette, qui instaura une "white night» entre 1983 et 1985. La formule n'est aujourd'hui plus en vigueur mais dans les faits, un groupe « blanc » est plus susceptible d'attirer les Cadiens dans un club zydeco.

Cette même crainte est perceptible dans le sens inverse chez les Créoles et semble d'ailleurs modeler leurs goûts : tandis que de nombreux jeunes Cadiens apprécient le zydeco, peu de Créoles manifestent un intérêt pour la musique cadienne et se rendent dans les festivals qui lui sont consacrés. Cette distance est d'autant plus justifiée que des incidents continuent de se produire lorsque des Noirs s'aventurent dans certains clubs cadiens, l'inverse n'ayant jamais provoqué d'incident. En 1995, l'entrée d'une salle de danse cadienne est refusée à une femme noire de Chicago, de passage pour une convention; à quelques mois d'intervalle, un homme du Kentucky subit la même discrimination lorsqu'il tente de se joindre au courir du Mardi Gras de Eunice. Victimes d'un code culturel qu'ils ignoraient, ces deux touristes ont révélé au grand jour des règles tacites alors dénoncées avec virulence par la presse locale et le milieu artistique et intellectuel. La première victime, qui s'avéra être procureur fédéral adjoint, mena à bien son procès qui entraîna la pose d'une plaque placée à l'entrée du club déclarant que le lieu est ouvert à tous, quelle que soit sa couleur. L'incident n'a pourtant pas transformé les mœurs, et la plaque a bien vite été recouverte d'affiches annonçant la programmation musicale, tandis que le public demeure exclusivement blanc. Un autre incident survint quelques années plus tard dans un club au Nord de Lafayette, dont le propriétaire refusa au groupe Balfa Toujours d'inviter au cours de leur concert leur ami à se joindre à eux. Menaçant de quitter la scène, le groupe eut gain de cause, et publia aussitôt une lettre dans l'hebdomadaire de Lafayette condamnant sévèrement cette attitude. Que la frontière ait été cette fois-ci franchie par des Louisianais et qu'il y ait eu dénonciation publique révèle en même temps une volonté du milieu artistique de faire évoluer les relations sociales de l'intérieur.

\section{«A quiet revolution»}

Depuis le milieu des années 80, le développement d'une politique touristique centrée sur le patrimoine franco-louisianais a suscité un engouement croissant pour la musique et la danse. Des groupes de fans effectuent de véritables pèlerinages dans les clubs locaux et à l'occasion de festivals. Les touristes blancs ont été les premiers à se rendre dans les clubs zydeco et contribuent ainsi à modifier la dynamique sociale des salles de danse, encourageant progressivement les Cadiens à suivre leur exemple. Originaire de Lake Charles, à l'ouest de l'Etat, Frances déménage à Lafayette dans les années 70. Dix ans plus tard, elle se rend pour la première fois dans un club zydeco sous l'impulsion d'une Québécoise. Elle s'éprend alors de cette danse et se met à transgresser les interdits de nombre de ses amis et de son club de danse. Frances s'est toujours sentie bien accueillie, des clients venant lui demander d'où elle venait, supposant qu'elle n'était 
pas du coin. Tout outsider se trouve ainsi souvent dans une position de médiateur, y compris l'anthropologue : je réalisai ainsi à plusieurs reprises que certains amis de plus de cinquante ans se rendaient avec moi pour la première fois dans un club zydeco, ma curiosité les incitant à surmonter leur réticence. Ils se montraient alors très soucieux d'adopter une attitude discrète et respectueuse pour ne pas faire intrusion, restant assis à une table plusieurs danses durant avant de se lancer sur la piste, observant avec attention les danseurs tout en leur demandant la permission de le faire.

Ces précautions sont caractéristiques des stratégies de négociations engagées par certains Cadiens, qui s'efforcent de faire évoluer les pratiques par le biais d'une action extrêmement progressive, tacite, sans revendication verbalisée comme c'est le cas dans le milieu artistique et intellectuel. Tel est le cas du propriétaire de Whiskey River Landing qui, en plus de ses tours en bateau sur le bassin de l'Atchafalaya, organise depuis 1997 un bal tous les dimanches après-midi. Située à côté du débarcadère, la salle de danse se trouve à quelques kilomètres du petit village d'Henderson, de l'autre côté de la levée (digue), au bord de l'eau. D'abord sans grands moyens, les bals ont lieu dans une petite salle sans air conditionné. Puis la salle s'agrandit, profitant de la réputation des groupes qui y jouent et en font la promotion dans tous les Etats-Unis. Le lieu figure désormais parmi les salles les plus prisées de la région, attirant plus de 300 personnes mêlant locaux et touristes américains et internationaux. Ami de Geno, le propriétaire lui fait part de son désir de le faire jouer, s'entretenant longuement avec lui sur les modalités de cette initiative. Le propriétaire du club fait d'abord venir le musicien seul, en tant que batteur au sein de Balfa Toujours, pour le rendre familier à sa clientèle. L'année suivante, il décide d'inviter le groupe du musicien certains samedis soir, lui consacrant stratégiquement un jour spécifique : ses clients savent ainsi à quoi s'attendre ce jour-là. Frances précise : «Avant ça, les Noirs n'allaient pas dans ce club. C'est loin, avec tous ces 'river rats', ils avaient peur. Alors tout le monde était un peu nerveux quand Geno est venu ». Le propriétaire de Whiskey River combine ainsi tous ses efforts pour agir de façon graduelle, afin d'éviter toute résistance qui réduirait ses efforts à néant.

"* Geno, le plus gros following que lui a c'est le monde blanc. Je veux pas faire quelque chose qui va casser mon business. As a businessman first, I've gotta keep my business, so I've gotta watch what I do. I've been called [menacé], but... I say Geno is a friend of mine, and I drop it like that. A Grant Street [club de Lafayette], ils ont après faire ça [depuis] des années. Quand tu vas sortir du village [ville] et venir dans la campagne, c'est pas la même affaire. Et je veux pas casser quelque chose qui peut-être dans deux ans va travailler. Je veux pas faire ça avant le temps [l'heure]".

Un an plus tard, en 2001, Geno Delafosse est programmé le dimanche, au même titre que les groupes cadiens.

Il arrive que certains journalistes viennent saper ces efforts en ne mesurant pas la complexité des relations entre Cadiens et Créoles. C'est ainsi que le Wall Street Journal, avide de rendre compte de l'attrait du zydeco auprès des touristes, consacre en 2001 un article à Frances : en dépit de ses efforts pour faire rencontrer au journaliste des Créoles, tout l'article se focalisa sur elle, suscitant l'amertume de certains Créoles à son égard. Si la presse extérieure est plus susceptible de commettre ce genre d'impair, les publications locales n'en sont pas à l'abris : dans sa page « attractions », l'édition 2004 de l'annuaire 
de la région de Lafayette définit ainsi le zydeco comme « un mélange de musique de danse cadienne et de blues africain $»^{24}$. Journaliste local spécialiste de zydeco, Herman Fuselier ne manque pas d'exprimer, dans un article diffusé à l'échelle nationale, son exaspération envers cette habitude persistante dans la presse même spécialisée de faire du zydeco une musique issue de la musique cadienne.

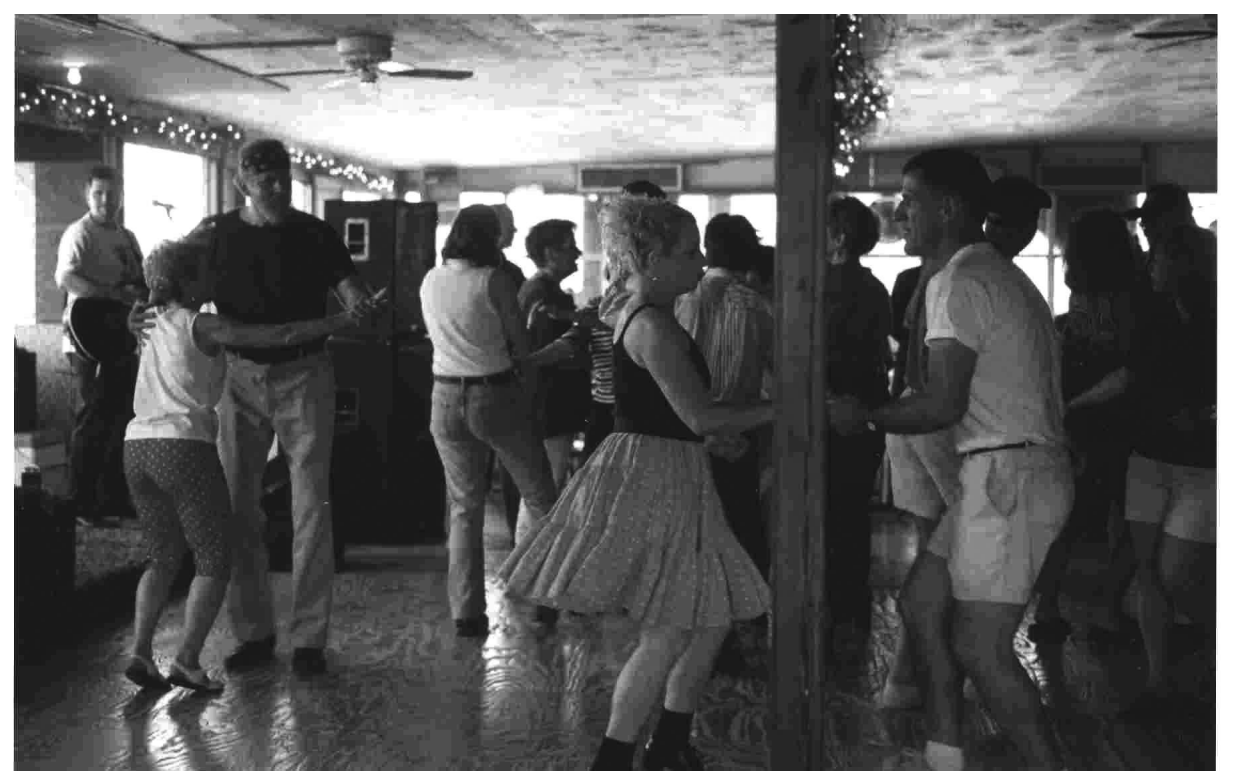

Photo 3 : Whiskey River Landing, un dimanche après-midi, Henderson, 2003 (C) Sara Le Menestrel).

La notion de temps d'adaptation et d'ajustement apparait incontournable dans l'évolution des relations sociales. C'est ce qui amène Don Cravins, animateur d'un programme de zydeco à la radio, à parler explicitement d'une "quiet revolution » montante, dont il compare le potentiel au mouvement des hippies dans les années soixante. Cette temporalité transparaît également sur un autre mode dans la façon dont les Créoles répondent aux tentatives de collaboration de certains Cadiens, qui s'efforcent depuis quelques années de les associer d'avantage au mouvement de renouveau identitaire et à des opérations touristiques. Leur longue mise à l'écart du renouveau francophone suscite toutefois une volonté d'indépendance et une résistance passive. Bien souvent, les Créoles tardent à répondre aux avances qui leur sont faites, demeurant en retrait. L'exemple de Dennis donne un éclairage particulièrement intense à cette attitude. Né à St Martinville, où les divisions sont aujourd'hui encore vivaces, il y grandit à une

24. C'est aussi la définition utilisée par Aaron Latham dans son article «Zydeco Fever in Lafayette » (New York Times, 30 mai 2004) ainsi que par plusieurs manuels et dictionnaires de musique. Même le CD Creole Bred, qu'Ann Savoy a conçu comme un hommage à la musique créole et zydeco, a reçu une critique dans le magazine Billboard, bible de l'industrie musicale américaine, sous le titre de "Cajun music gets its due » (Fuselier, 2004). 
époque particulièrement tendue, les années 60-70. Orphelin d'un père ouvrier dès l'âge de dix ans, il accumule les petits travaux après l'école pour soutenir sa mère avec ses frères, tout en s'inscrivant au programme artistique pour réaliser son rêve, être peintre. L'enseignante lui fait rapidement comprendre " qu'il n'est pas fait pour l'art» et brise tous ses espoirs. Alors âgé de seize ans, effondré - «c'était comme un deuil »-il décide de s'engager dans l'armée. Raffermi par son expérience de l'étranger et ses rencontres, il prend la décision de s'installer dans son village natal en dépit de la pesanteur du climat social. Il développe ses talents musicaux, est engagé dans le groupe zydeco de son frère, aujourd'hui de réputation internationale, et exerce ses talents de peintre en autodidacte. Aujourd'hui, alors qu'il participe à des expositions à l'échelle nationale, Dennis ne fait pas la promotion de son travail en Louisiane. Aller voir ses oeuvres requiert une certaine persévérance : beaucoup d'habitants de St Martinville disent ne pas le connaître, tandis que son atelier a l'allure d'une maison non habitée et arbore le signe " No Trespassing ». S'il affirme sa détermination en ayant fait le choix de ne pas se laisser évincer, le déni de son talent l'a conduit à laisser les gens venir à lui.

"J'ai créé une audience à l'intérieur de moi. Alors je n'ai pas besoin de [il applaudit], je n'ai pas besoin de ça. Il y a une voix en moi qui me répète de rester ici (...). Alors j'ai du apprendre à cultiver un univers à l'intérieur de moi. Et c'est une bonne chose parce que je ne me suis jamais mis en colère. C'est ce qui m'aide à garder ma raison. Je pense souvent au Mahatma Gandhi, il a dit quelque chose qui m'aide à aller de l'avant bien souvent, quand un défi se présente à moi. Il a dit: 'notre force ne vient pas de nos capacités physiques, mais d'une volonté indomptable'».

Aux discriminations subies en tant que Noirs s'ajoute chez certains Créoles l'amertume provoquée par la surenchère cadienne, qui les incite à se replier dans une victimisation que d'autres membres du groupe leur reprochent : ceux qui sont engagés dans la promotion de leur culture revendiquent au contraire une attitude réactive au lieu d'être défensive. La prise en main de cette promotion passe bien souvent par une volonté ferme de ne pas être assimilés aux Cadiens ${ }^{25}$. Dès 1988, le producteur du musicien Buckwheat Zydeco, fervent défenseur de la spécificité créole, formule un contrat sans ambiguïté : «N'utilisez pas le mot 'Cajun' pour promouvoir ce spectacle. Cela est formellement interdit et annulera le présent contrat. Appeler Buck un Cadien c'est comme si on appelait un Irlandais un Anglais, et se référer au zydeco comme de la musique cadienne, c'est comme si on appelait le reggae du calypso » (Olivier, 1999 : 122). Animateur d'une émission de radio sur le zydeco, John revendique quant à lui le droit des Créoles à contrôler leur patrimoine musical :

"On préfère conserver le zydeco comme c'est, et ne pas laisser quelqu'un nous dicter ce que ça devrait être. Laissons la race qui a créé cette musique la promouvoir, la soutenir, laissons-la dans ce cadre, ne laissons pas quelqu'un être le porte parole ou l'expert. Il faut vivre cette culture pour pouvoir la connaître. Je ne peux pas parler du cadien, parce que je n'ai pas vécu dans cette société ».

25. De là vient la création du Creole Music Awards, pendant du Cajun Awards attribué par la CFMA. 
Sa détermination l'amène ainsi à fonder son argument sur une différence " raciale », confondue ici avec la culture. John n'en revendique pas moins la multiplicité de ses ascendances, même en public :

"*Parce que moi je suis mêlé avec les trois races [noire, blanche, amérindienne]. Je suis intéressé pour faire tout le monde connaître c'est pas de différence, parce que je suis juste resté dessus le four plus longtemps que les Blancs, c'est tout ! ».

C'est donc l'identité noire des Créoles qu'il privilégie pour légitimer leur prérogative sur la définition du zydeco. Celui-ci apparaît comme un enjeu politique du à sa popularité nationale grandissante (si bien qu'elle dépasse aujourd'hui celle de la musique cadienne) et son pouvoir d'attraction auprès des touristes, qui amène John à mobiliser d'autres catégories d'appartenance : en mettant l'accent sur la distinction Blanc/Noir, il tient à se prémunir de toute « récupération » des Cadiens au nom d'un héritage musical commun et à s'assurer qu'ils en récolteront les bénéfices économiques ainsi qu'une hausse de statut et de pouvoir. Ce souci de distinction n'est toutefois pas incompatible avec la revendication de valeurs communes.

\section{Respectabilité et ruralité}

Dans le Sud-Ouest, une majorité de Créoles comme de Cadiens travailla comme métayer après l'abolition de l'esclavage. Ce contexte économique les amena à être en concurrence, mais aussi à valoriser leur caractère laborieux et leur lutte quotidienne contre l'adversité ${ }^{26}$. Ce facteur (occupations communes, travail commun) est indissociable de l'importance fondamentale accordée à la notion de respectabilité, qui transparaît très clairement dans les appréciations musicales. Elle s'exprime par un souci aigu du comportement et de l'apparence des musiciens ${ }^{27}$.

La politesse et la propreté des musiciens sont constamment invoquées pour appuyer un jugement favorable: on insiste sur leur tenue impeccable, sans que cela implique une recherche vestimentaire, mais simplement en référence au soin apporté (jeans et chemise boutonnées, propres et repassés). Pour exprimer sa désapprobation du style très rock de Wayne Toups, un interlocuteur cadien invoque son débardeur échancré laissant apparaître son buste, ses cheveux long, en bataille, et sa barbe de trois jours, qui lui "font honte ». L'attention envers le public est elle aussi particulièrement valorisée : rester accessible quelle que soit sa notoriété, montrer tous les égards à ses fans, leur sourire, les saluer, donner de soi sans lassitude ni arrogance avec un souci constant d'affabilité. C'est l'ensemble de ses facteurs qui définit la capacité à « bien se présenter », à « soigner

26. Cette proximité sociale suscite des pratiques d'entraide, comme la boucherie (où l'on tuait et partageait le cochon), qui ne sont toutefois pas toujours interprétées comme un signe de solidarité mais aussi associées à la nécessité : "Il fallait manger! Je ne sais pas si on voulait aider qui que ce soit, on le faisait juste parce qu'on avait faim et le cochon était là !"».

27. A. Anthony souligne cet aspect pendant la ségrégation à La Nouvelle-Orléans : « The emphasis that was placed on appearances was in keeping with the Creole sense of distinctiveness. Not only were they different from others : they looked different as well » (1978: 122). 
son monde » et fonde la notion de respectabilité ${ }^{28}$. La réception du musicien créole Geno Delafosse parmi les Cadiens illustre l'impact déterminant de ce mode d'être. Tous ses fans insistent sur sa gentillesse, son soin à venir leur serrer la main. Le propriétaire de la salle de danse cadienne qui l'a recruté justifie ainsi son choix :

"*Geno est respectable lui-même. Il te traite comme t'es supposé d'être traité. He'll talk to you politely, he's clean, he ain't into no shit ».

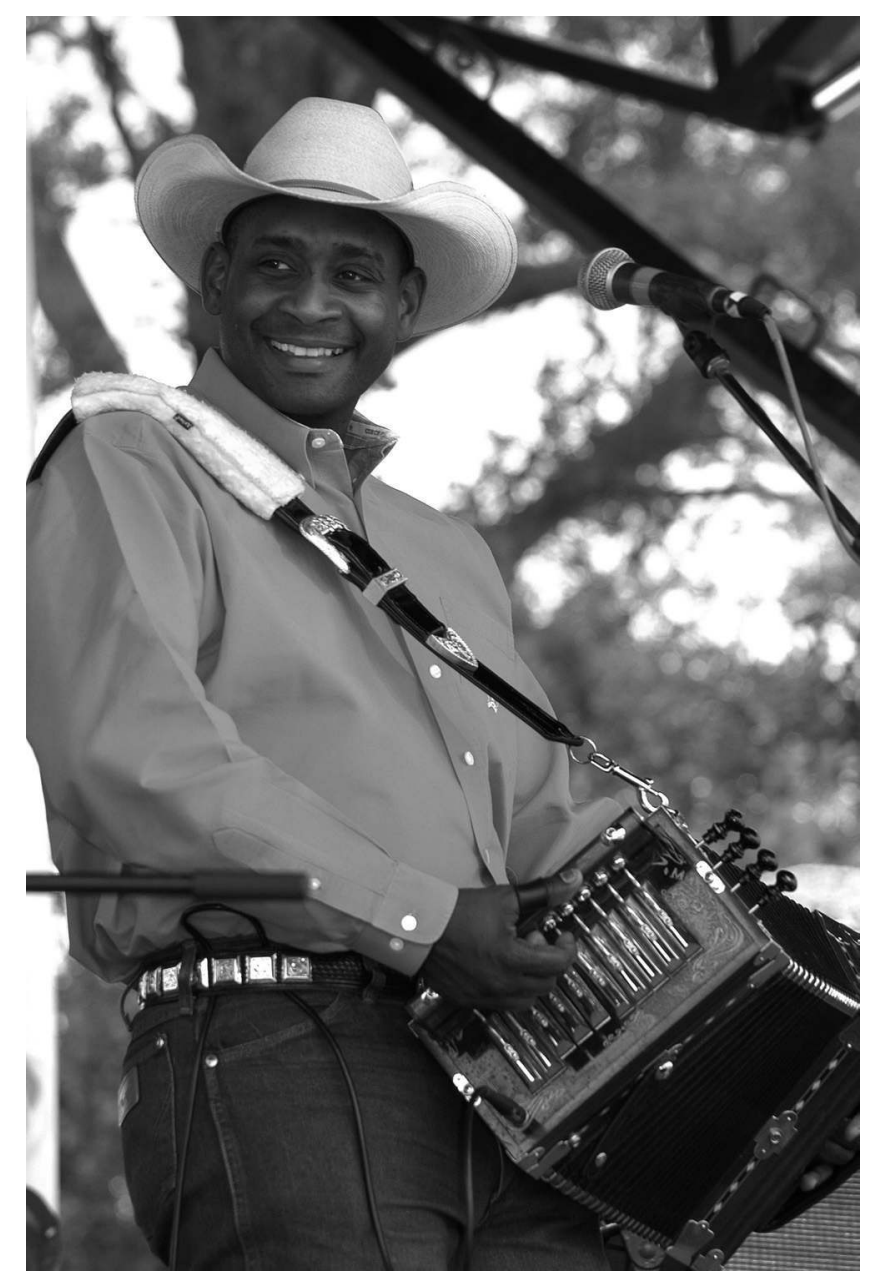

Photo 5 : Geno Delafosse, Crawfish Festival, Breaux Bridge, 2004

(C) David Simpson, LSUE Office of Public Relations).

28. L'importance de cette notion s'inscrit notamment dans l'héritage de Booker T. Washington et de son action pour rendre aux Noirs leur dignité et les hausser socialement au rang des Blancs en particulier par le biais de l'éducation. S. Smith témoigne de l'impact de cette perspective dans le domaine artistique au travers de la politique du producteur de disques noir américain Motown, qui investit beaucoup d'énergie à soigner l'image publique de ses artistes, leur apparence, leur diction, selon ses critères de respectabilité (1999: 121). 
Fils d'un fermier lui-même musicien renommé, vivant dans une ferme où il élève chevaux et bétail, francophone, Geno Delafosse incarne toutes les vertus associées à l'environnement rural et aux travaux agricoles, opposées au modèle de l' «urban bad guy ». Repoussoir associé aux oisifs, quémandeurs et fainéants que seraient les jeunes des milieux urbains souvent dénommés "hippies », cette figure est invoquée comme l'antithèse des notions d'ordre et de respectabilité. «Il faut être propre. J'aime pas ces foutus hippies, avec un foulard sur la tête, une boucle d'oreille, les cheveux dans la figure. Ils me mettent en colère. Moi je mets un chapeau de cow-boy partout où je vais. Ca me vient d'avoir monté à cheval, d'avoir entraîné des quarts et des purs-sangs ",, déclare explicitement Boozoo Chavis (Sandmel et Olivier, 1999 : 79), qui a enregistré le premier hit zydeco en 1954, I've got a paper in my shoe. Son discours était assorti de choix musicaux et vestimentaires qui contrastaient avec l'autre «père fondateur » du zydeco, C. C. Chenier : «Car si Chenier rappelait toujours à son auditoire qu'il venait de la campagne, Chavis faisait bien savoir qu'il ne l'avait jamais quittée. Par contraste avec le costume cravate impeccable de Chenier, Chavis faisait ses déclarations coiffé d'un Stetson et d'un tablier en plastique (pour empêcher la sueur de mouiller les soufflets de l'accordéon). Et tandis que Chenier jouait le gros accordéon piano, Chavis gardait son vieil accordéon français à une ou trois rangées » (Tisserand, 1998 : 293). Même si l'un comme l'autre étaient fils de métayers, leur itinéraire est contrasté : Clifton est parti travailler comme ouvrier dans l'industrie pétrolière à Lake Charles, puis à l'est du Texas à partir de 1946, d'où il a engagé une carrière internationale, prenant très à cœur son rôle d' « ambassadeur du zydeco ». Boozoo était éleveur de chevaux de course; il a abandonné sa carrière musicale au début des années 1960, s'estimant spolié par les compagnies de disques, puis ressurgit triomphalement en 1984 jusqu'à sa mort en 2001. Toutefois, en dépit de leurs orientations différentes, l'un comme l'autre ont été les principales sources d'inspiration des générations suivantes de musiciens zydeco.

La revendication de cet héritage par les interprètes de « nouveau zydeco », style issu des années 1980, ne les préserve pas des critiques de leurs détracteurs, qui invoquent leur influence rap et hip hop. Invoquées par certains experts locaux, les justifications musicales de ce lien semblent pourtant d'avantage relever d'un souci d'inscrire le nouveau zydeco dans la modernité et la musique de masse, soulignant par là même son potentiel et son envergure ${ }^{29}$. Du reste, le style vestimentaire de ces musiciens ne reflète pas cette association : leur tenue demeure décontractée et sobre (tee-shirt ou chemises 'sport' plus ou moins colorées, jeans, casquettes), comme beaucoup de musiciens cadiens et zydeco, et ne rejoint pas le style plus ostentatoire de C.C. Chenier ou Buckwheat Zydeco (lunettes noires, bijoux, accessoires, accordéon brillant...), plus proche des joueurs de blues. Les couvertures de CD sont éventuellement l'occasion d'excentricité, comme le dernier

29. B. Sandmel présente comme typiques du nouveau zydeco sa forte ligne de basse et un rythme syncopé extrêmement populaire auprès des danseurs : le "double-clutching ", consistant en deux frappes sur la grosse caisse sur le modèle d'un battement de cœur, ainsi que des chœurs similaires au rap (1999: 152). Musicalement, le son, le rythme sont accentués (grosse caisse), certains phrasés systématisés, mais la base demeure très semblable à celle du zydeco «traditionnel ». Certains témoignages de musiciens ont sans doute concourus à l'association entre rap et zydeco : Beau Jocque explique dans son CD Beau Jocque Boogie (1994) qu'il s'est inspiré de la chanson du rappeur FM «Gimme what you got (for a porkchop) 》 pour son hit « Give him cornbread». 
album de Keith Franck où il est revêtu d'un haut et d'un chapeau en fausse fourrure de style panthère, ou Beau Jocque photographié en costume cravate. Leurs opposants ne font d'ailleurs jamais référence à leur tenue mais dénoncent l'apport du rap associé à un stéréotype négatif et accusé de dénaturer le zydeco, arguant également de la pauvreté des paroles pour justifier leur jugement. Les représentants du style créole, en particulier la famille Ardoin, mettent un point d'honneur à ne pas être identifiés avec les musiciens de zydeco actuels, insistant sur l'origine rurale de leur style. La prégnance de cet imaginaire amène à opposer les appréciations des Créoles urbains et des Créoles ruraux :

"*Y a deux Créoles : le gros créole et le créole petit. Le gros créole, eusses l'aime pas la musique de zydeco, eusse l'aime le jazz ou quelque chose comme ça. Mais le petit créole, comme nous autres, eusses l'aiment la musique de zarico »,

déclare Tim, sans tenir compte des stratifications sociales au sein même de ces deux groupes et de la popularité du jazz dans la Louisiane rurale du début du $20^{\mathrm{e}}$ siècle.

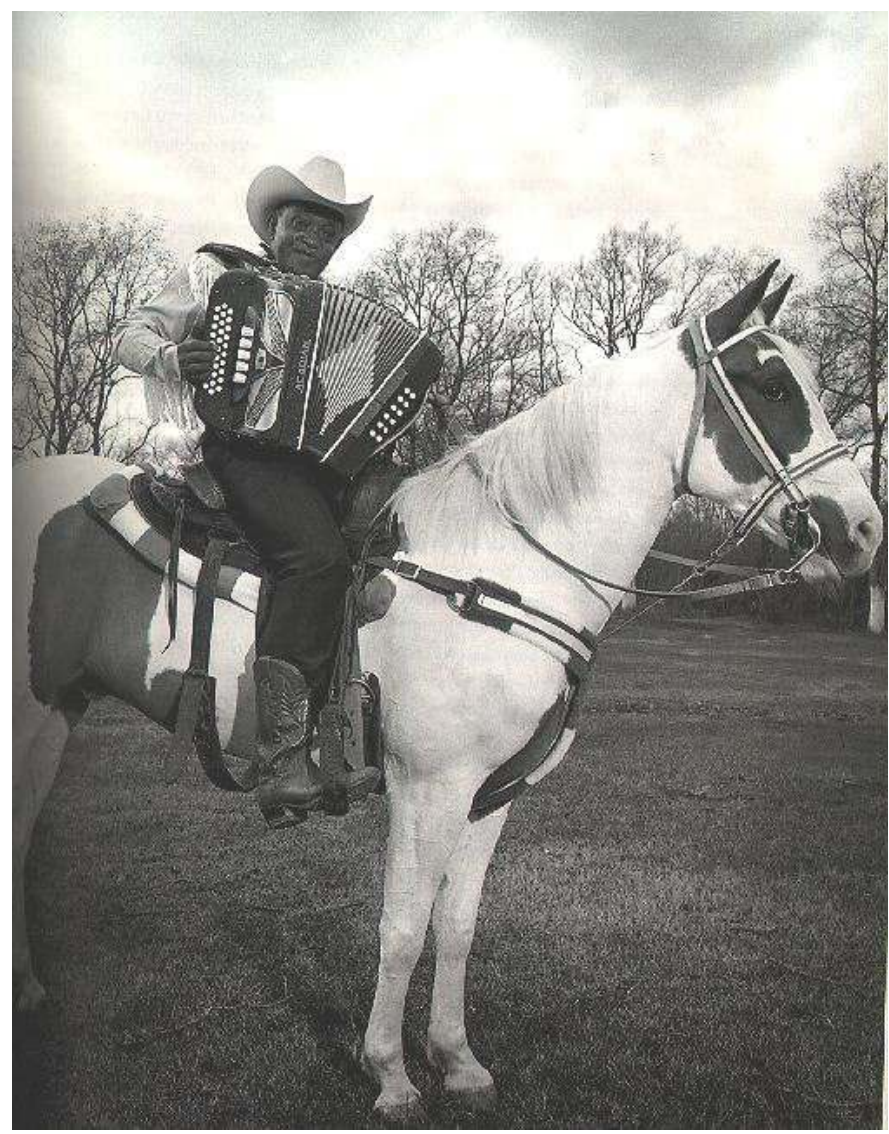

Photo 6 : Boozoo Chavis (C Rick Olivier). 
Cette image consensuelle passe sous silence tout un pan de l'histoire de cette musique, suscitant la production d'une mythologie régionale fortement induite par les phénomènes de migration. Le zydeco est indissociable de la migration des Créoles, à partir de la seconde guerre mondiale, dans les grands centres industriels du sud-est du Texas, pour y travailler dans l'industrie pétrolière. C'est donc en milieu urbain que s'est opérée la combinaison du style créole et du rythm and blues ${ }^{30}$. Et c'est au Texas que le zydeco a émergé comme genre à part entière, se trouvant intégré dans un réseau commercial qui a contribué à son essor (Minton, 1996; Wood, 2001).

\section{Stratégies de reconnaissance et choix musicaux}

La mise en avant de l'origine rurale et spécifiquement louisianaise, combinée à celle de l'attachement à la famille, conduit à une attitude ambivalente envers la renommée nationale et internationale, de la part des Créoles comme des Cadiens. Les titres honorifiques (Grammies, National Awards) sont valorisés, certains ayant même suscité des conflits de pouvoir, comme celui de «King of zydeco » parmi les musiciens créoles. L'attrait pour le succès commercial et l'intégration à un marché national est d'autant plus prononcé chez ces derniers, qui éprouvent un besoin de reconnaissance et de validation à la mesure des discriminations qu'ils rencontrent en tant que Noirs. La revendication d'une identité spécifique est de surcroît plus récente chez les Créoles, qui dans les années 1960 étaient avant tout préoccupés par la défense de leurs droits civiques. La diffusion du zydeco dans le réseau médiatique représente un moyen d'accroître leur capacité d'action et leur pouvoir économique. Le rayonnement de Buckwheat Zydeco (Stanley Dural, né en 1947) est à ce titre exemplaire : il a été nominé quatre fois pour le Grammy Award, compte à son actif une dizaine de publicités (Coca Cola, Cheerios, Budweiser, etc...), la cérémonie de fermeture des jeux olympiques de 1996 à Atlanta, l'inauguration présidentielle de Bill Clinton en 1997, et des enregistrements avec Eric Clapton, Keith Richards, Los Lobos... Ses compositions rendent hommage à C.C. Chenier, dont il a été l'organiste pendant trois ans, mais emprunte aussi au rock, au funk, au rythm and blues, et à la soul. Buckwheat a réinterprété des chansons de grandes stars telles que Hey, Good Lookin' de Hank Williams ou Beast of Burden des Rolling Stones. Beaucoup de musiciens de zydeco réinterprètent des grands nom du rock, de la soul, du rythm and blues et même du reggae (notamment BB King, Sam Cooke, Bob Marley) provoquant le ralliement d'un auditoire blanc féru de ces figures emblématiques de la musique populaire américaine, qui leur permet ainsi d'élargir le cercle de leurs fans et d'accroître leur reconnaissance.

Le producteur de Buckwheat exprime bien l'enjeu d'une telle démarche : les rubriques « Cajun », « Blues » ou « Country » restent reléguées au fond des magasins de disques. Puisque Bob Marley et les Gipsy Kings sont classés « rock'n'roll », il considère que lui aussi peut demander à être classé dans ce rayon (Tisserand, 1999 : 208-10) ${ }^{31}$. Le succès commercial apparaît ainsi comme un moyen de promouvoir le statut du zydeco. Que les interprètes de nouveau zydeco revendiquent l'inspiration de la chaîne MTV relève bien

30. Le terme « zydeco » apparaît d'ailleurs sous une variante écrite dans les enregistrements commerciaux de Noirs non créoles de Houston, à commencer par le joueur de blues Lightnin' Hopkins, avec Zolo Go (1949), où figurent des références au zydeco et dans lequel un orgue imite un accordéon (Minton, 1996).

31. Les répercussions de cette stratégies se font sentir localement, Buckwheat se produisant sur la scène principale du Jazz Fest de La Nouvelle-Orléans (l'un des plus grands festivals des Etats-Unis) et non sur la scène Fais DoDo consacrée aux groupes cadiens et zydeco. 
de cette même démarche. L'un des représentants les plus populaires du nouveau zydeco, Keith Frank, a composé le générique de la série télévisée grand public The Jeffersons, faisant de cette chanson, Movin'on up, un succès qui fait toujours l'unanimité parmi les danseurs, en combinant style créole et soul. Le fait qu'une majorité de musiciens de zydeco chantent aujourd'hui en anglais s'inscrit dans cette même stratégie et éclaire là encore le contraste avec les musiciens cadiens, qui chantent avant tout en français. Pour les Créoles, le français est encore perçu comme un stigmate supplémentaire ${ }^{32}$. Leur position socio-économique au plus bas de l'échelle sociale louisianaise les incite à renoncer aux critères objectifs d'appartenance tels que la langue ${ }^{33}$ et à tout mettre en œuvre pour conquérir un large auditoire. Cette stratégie leur confère une hausse de statut et un prestige social en tant qu'Africains Américains, qui déborde la spécificité créole. Elle n'est pas sans évoquer le rôle de leaders politiques et d'élite sociale que de nombreux Créoles de couleur ont joué parmi les Noirs louisianais, à partir de la Reconstruction. L'enjeu politique que représente le zydeco n'est pas perceptible au travers des chansons ou de discours revendicatifs, mais dans les choix musicaux des musiciens, dans lesquels intervient une démarche commerciale. La carrière politique de Don Cravins l'illustre bien, son succès étant étroitement lié à son implication dans la promotion de cette musique, qui lui a valu le surnom de «Zydeco man ».

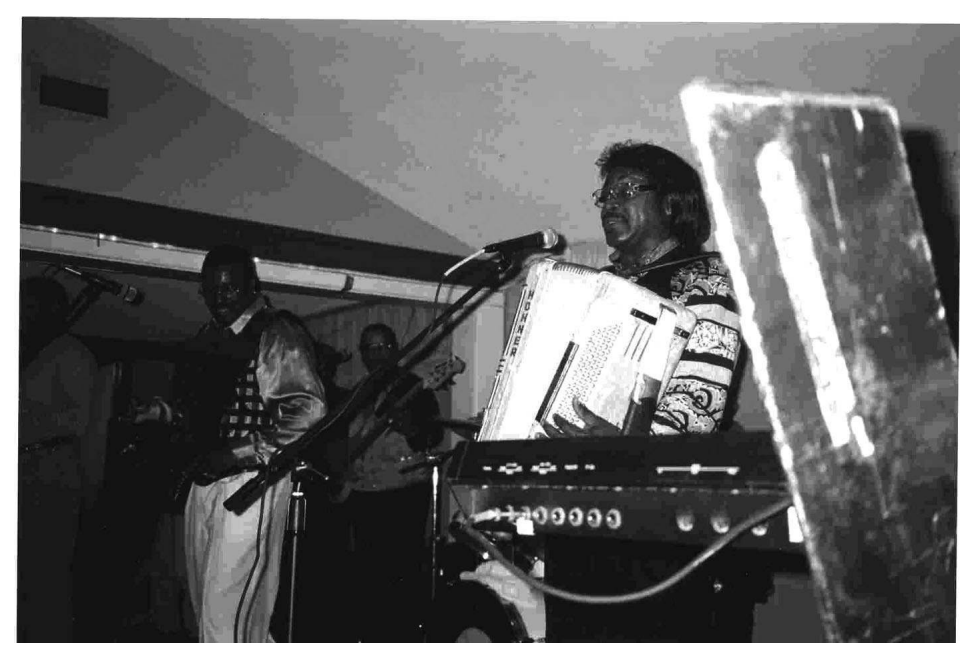

Photo 7 : Buckwheat Zydeco, El Sid-O's Zydeco and Blues Club, Lafayette, 2001 (C) Sara Le Menestrel)

32. Tandis qu'un certain nombre de parents cadiens non francophones tient à rétablir la transmission du français, bien rares sont les enfants créoles qui sont inscrits dans les programmes bilingues d'immersion française offerts dans les écoles primaires et secondaires du Sud-Ouest louisianais. Le nombre de Louisianais se déclarant d'ascendance créole (3 291) dans le recensement de 2000 reste bien en deçà des estimations plus fines. La combinaison de «l'identification raciale » (noir) et de la pratique du français (standard, cadien ou créole) aboutit à 30295 Noirs francophones, ce qui reste toutefois une faible proportion (15\%) sur le total de 198000 francophones recensés en 2000 (Henry 2004, à paraître).

33. Précédés par les Blancs non-cadiens, puis les Cadiens et les Noirs non-francophones, les Créoles sont ceux dont le revenu moyen par foyer (19 400\$) et le pourcentage de diplômés universitaires $(6,2 \%)$ est le plus faible, et ils sont les plus représentés parmi les ouvriers $(23,2 \%)$ et les employés $(35,8 \%)$. 
Les efforts de visibilité des musiciens de zydeco ne sont pourtant pas systématiques, un bon nombre d'entre eux ne cultivant pas la promotion de leur musique hors des frontières louisianaises. L'accordéoniste zydeco Rockin' Dopsie, qui s'est fait remarquer par sa prestation avec Paul Simon sur l'album Graceland, n'a pourtant pas manifesté le souhait d'en recueillir toutes les répercussions : il a ainsi décliné une invitation à jouer dans la très célèbre émission télévisée Saturday Night Live, répondant qu'il s'était engagé pour un concert dans une fête paroissiale. Dans un autre registre, D.L. Ménard, baptisé le " Cajun Hank Williams », et nommé " ambassadeur de la musique cadienne » par son village natal, a été nominé comme "meilleur album traditionnel » des Grammies en 1993 et a reçu l'année suivante le National Heritage Fellowship Award du National Endowment for the Arts des mains de Hillary Clinton. Sa carrière internationale ne l'a pourtant pas amené à transformer son cadre de vie : il a conservé la même petite maison, modeste, dans le village où il est né, poursuivant son activité de fabriquant de chaises en bois. Il y a quelques années son atelier, qui n'était pas assuré, a été dévasté par un incendie. C'est grâce au bénéfice (collecte d'argent) organisé par ses proches qu'il a pu le reconstruire.

Les différentes attitudes des musiciens vis-à-vis de leur rayonnement national et international ne correspondent pas nécessairement à des choix personnels divergents, mais entretiennent une relation dialectique. Issu de plusieurs générations de musiciens, le jeune Keith Frank a sorti en 2001 un CD avec son oncle, Creole Connection, faisant honneur à l'ancien répertoire français sous le nom de groupe The Masked Band, jouant ainsi sur l'effet de surprise que pouvait susciter ce choix musical et exprimant par là même son caractère polémique : le port de masques sur la photo de l'album suggérait que Keith Franck devait se travestir pour interpréter des chansons communes aux Cadiens, et qui ne sont donc pas forcément jugées légitimes ${ }^{34}$. Quoiqu'il en soit, la combinaison de musique française et de nouveau zydeco dans l'album traduit un souci de concilier héritage « traditionnel» et style contemporain. La volonté de hausser le statut de la musique franco-louisianaise en l'adaptant aux critères de la culture de masse est combinée à une forte conscience de la localité ${ }^{35}$. Même ceux qui vivent avant tout de leurs tournées hors de l'Etat sont toujours soucieux de rendre hommage aux musiciens pionniers et d'être reconnus dans leur région natale - bien que les goûts de ce public et son exigence ne soient pas toujours en accord avec la réception nationale. La relative sobriété vestimentaire semble s'inscrire dans cette même démarche : respecter une certaine norme dans l'attitude et l'apparence sans restreindre l'éventail des choix et de la créativité musicale.

\section{Conclusion}

Le domaine musical constitue en Louisiane un espace de négociations, voire de contestations, dans lequel la transgression de la ligne de couleur est risquée, et même aujourd'hui revendiquée par des musiciens, des producteurs, des propriétaires de

34. Les Créoles qui se consacrent au répertoire français, dit « style créole », sont d'ailleurs très peu nombreux et leurs enregistrements restent très marginaux par comparaison avec le succès des musiciens de zydeco. On peut ainsi citer Bois Sec Ardoin, Eddie Poulard et D’Jalma Garnier, et parmi la jeune génération Joe Hall, Dexter Ardoin et Cédric Watson.

35. R. Sexton met en lumière l'importance de ce sens de la localité dans l'identité cadienne (2000b). Cet attachement est d'ailleurs reflété par les statistiques, puisque les Cadiens représentent le groupe le plus sédentaire des Etats-Unis (Henry, 2004, à paraître). 
clubs, des danseurs, guidant leur choix musicaux et offrant une vision alternative de la culture franco-louisianaise. Ces stratégies n'amoindrissent pas la complexité des rapports sociaux entre Cadiens et Créoles ni la portée de certains clivages et d'identités diasporiques (acadienne, noire ${ }^{36}$ ), même si elles sont toujours combinées à d'autres critères d'appartenance qui témoignent de la fluidité des représentations identitaires locales. La collaboration d'A. Ardoin et de D. McGee a vraisemblablement été sinon exceptionnelle, du moins très marginale par rapport à la norme, surtout sur scène où les sanctions de cette transgression pouvaient être fatales, comme en témoigne le sort d'A. Ardoin. Par ailleurs, la ligne de couleur n'est pas le seul facteur en jeu dans l'interaction entre Cadiens et Créoles, dans laquelle interviennent des hiérarchies sociales issues des ramifications des identités franco-louisianaises. Les tentatives de réappropriation des traditions musicales ne relèvent ainsi pas d'une opposition binaire fondée sur l'origine « ethnique » (cadienne/ créole) mais s'opèrent aussi bien au sein des deux groupes.

La volonté de plus en plus verbalisée par les universitaires et les artistes de valoriser les correspondances entre musique cadienne, créole et zydeco ne va pas sans ambivalence, puisqu'elle se double souvent d'une légitimation de leur différence. Cette perception «racialisée » de la musique locale, si elle émane de Noirs comme de Blancs, est plus souvent revendiquée et explicitée publiquement par ces derniers, qui se prémunissent ainsi de toute accusation d'appropriation par les Blancs de la musique créole. Les paroles d'Ann Savoy lors du Jazz Fest de 2004 l'illustrent bien. Interviewée aux côtés de l'accordéoniste créole Geno Delafosse, qui figure dans le CD Creole Bred qu'elle a produit, elle déclare : « Je tenais à montrer que ce sont deux musiques différentes, de deux groupes francophones différents ». Elle ajouta qu'aucun Cadien n'interprétait du zydeco dans son disque, associant implicitement cette démarche à une imposture. Le sourire aux lèvres, Geno Delafosse s'étonna de cette distinction insistante. Les représentations et l'évolution de la musique franco-louisianaise sont précisément modelées et circonscrites par ces ambiguités et ces paradoxes.

36. Comme on l'a vu, la revendication d'une appartenance à la diaspora noire n'implique pas nécessairement la reconnaissance d'une ascendance africaine. 


\section{Références bibliographiques}

ANCELET, Barry Jean,

1989. Cajun Music : Its Origins and Development. Lafayette, Center for Louisiana Studies : University of Southwestern Louisiana.

1992. «Introduction », in Philip Gould, Cajun Music and Zydeco, pp. ix-xxi. Baton Rouge : Louisiana University Press.

1996. «Zydeco/Zarico : The Term and the Tradition », in James H. Dormon, ed., Creoles of color of the Gulf south, pp. 126-143. Knoxville : University of Tennessee Press.

Anthony, Arthe Agnes, 1978. The Negro Creole Community in New Orleans, 1880-1920 : An Oral History. Thesis (Ph.D.) : University of California at Irvine.

Bell, Caryn Cossé, 1997. Revolution, Romanticism, and the Afro-Creole Protest Tradition in Louisiana, 17181868. Baton Rouge : Louisiana State University Press.

Bernard, Shane K., 1996. Swamp Pop : Cajun and Creole Rhythm and Blues. Jackson : University Press of Mississippi.

Brasseaux, Carl A., Keith P. Fontenot et Claude F. Oubre, 1994. Creoles of Color in the Bayou Country. Jackson : University Press of Mississippi.

Broven, John, 1983. South to Louisiana : the Music of the Cajun Bayous. Gretna, La. : Pelican Pub.

Dole, Gérard, 1995. Histoire musicale des Acadiens, de la Nouvelle-France à la Louisiane : 1604-1804. Paris : L'Harmattan.

Fairclough, Adam, 1995. Race \& Democracy: The Civil Rights Struggle in Louisiana, 1915-1972. Athens : University of Georgia Press.

FuSELIER, Herman, 2004. «Zydeco is not Cajun », Zyde-zine, revue électronique, part I, http://www.homestead. com/cajunzydeco/files/articles/a040707demi.htm.

Henry, Jacques, 2005 (à paraître). « Pourquoi les Cadiens disparaissent et les Créoles restent invisibles » in $L a$ Louisiane, Guy Clermont (éd.), Limoges : Presses Universitaires de Limoges.

Hughes, Michael, Hertel R. Bradley, 1990. « The Significance of Color Remains : A Study of Life Chances, Mate Selection, and Ethnic Consciousness Among Black Americans », Social Forces, 68 (4), pp. 11051120 .

KLINGLeR, Thomas A., 2004. « Beyond Cajun : Towards an Expanded View of Regional French in Louisiana », communication présentée au colloque « Language Variety in the South III », 16 avril 2004. Tuscaloose : University of Alabama.

Le Menestrel, Sara,

2006 (à paraître). « Métissage, créolisation : usages et instrumentalisation », in Actes du colloque, « Les Français aux Amériques : bilans et perspectives », 12 décembre 2002. Paris : Ecole des hautes études en sciences sociales.

2005 (à paraître). «Déclinaisons et enjeux de l'identité créole en Louisiane », in Actes du colloque, « Situations créoles : pratiques et représentations », Université du Québec à Montréal, 29-30 mai 2003. Québec : Editions Nota Bene.

1999. «A la croisée des regards: la construction du patrimoine franco-louisianais », Ethnologie française, 29 (3), pp. 409-419.

1999. La voie des Cadiens. Tourisme et identité en Louisiane. Paris : Belin, Collection « Cultures américaines ».

Minton, John, 1996. « Houston Creoles and Zydeco : The Emergence of an African American Urban Popular Style », American Music, 14 (4), pp. 481-525. 
French music, Cajun, Creole, Zydeco : ligne de couleur et hiérarchies sociales dans la musique franco-louisianaise

SAndmel, Ben et Rick Olivier, 1999. Zydeco ! Jackson : University Press of Mississippi.

SAvoy, Ann Allen, 1984. Cajun Music : A Reflection of a People. Eunice, La. : Bluebird Press.

SEXTON, Rocky,

2000a. «Zydeco Music and Race Relations in French Louisiana », in Peter Kivisto \& Georganne Rundblad, eds, Multiculturalism in the United States, pp. 175-184. Thousand Oaks, CA : Pine Forge Press.

2000b. «"You Might Be a Cajun if..." : The Tenacity of Place in a Changing World», in Jim Norwine \& Jonathan Smith, Worldview Flux : Perplex Values among Postmodern People, pp. 111-132. Lanham: Lexington Books.

Smith, Suzanne E., 1999. Dancing in the Street : Motown and the Cultural Politics of Detroit. Cambridge : Mass., Harvard University Press.

Snyder, Jared M., 1997, «Squeezebox: The Legacy of the Afro-Mississippi Accordionists », Black Music Research Journal, 17 (1), pp. 37-57.

SonNIER, Austin M., 1989. Second linin': Jazzmen of Southwest Louisiana, 1900-1950. Lafayette : Center for Louisiana Studies, University of Southwestern Louisiana.

Thompson, Shirley, 2001. «'Ah Toucoutou, ye conin vous': History and Memory in Creole New Orleans », American Quarterly, 53 (2), pp. 232-265.

Tisserand, Michael, 1998. The Kingdom of Zydeco. New York: Arcade Pub.

Ware, Vron et Les Back, 2002. Out of Whiteness : Color, Politics, and Culture. Chicago : University of Chicago Press.

Wood, Roger, 2001. « Southeast Texas : Hot House of Zydeco », The Journal of Texas Music History, 1 (2), pp. 23-45.

WADE, Peter, 2000. Music, Race \& Nation : Musica Tropical in Colombia. Chicago : University of Chicago Press. 
